Article

\title{
Enantioselective Benzylation and Allylation of $\alpha$-Trifluoromethoxy Indanones under Phase-Transfer Catalysis
}

\author{
Yumeng Liang ${ }^{1}$, Mayaka Maeno ${ }^{1}$, Zhengyu Zhao ${ }^{1}$ and Norio Shibata ${ }^{1,2, *(D)}$ \\ 1 Department of Nanopharmaceutical Sciences, Department of Life Science and Applied Chemistry, \\ Nagoya Institute of Technology Gokiso, Showa-ku, Nagoya 466-8555, Japan \\ 2 Institute of Advanced Fluorine-Containing Materials, Zhejiang Normal University, 688 Yingbin Avenue, \\ Jinhua 321004, China \\ * Correspondence: nozshiba@nitech.ac.jp; Tel./Fax: +81-52-735-7543
}

Received: 5 July 2019; Accepted: 29 July 2019; Published: 30 July 2019

\begin{abstract}
The organo-catalyzed enantioselective benzylation reaction of $\alpha$-trifluoromethoxy indanones afforded $\alpha$-benzyl- $\alpha$-trifluoromethoxy indanones with a tetrasubstituted stereogenic carbon center in excellent yield with moderate enantioselectivity (up to 57\% ee). Cinchona alkaloid-based chiral phase transfer catalysts were found to be effective for this transformation, and both enantiomers of $\alpha$-benzyl- $\alpha$-trifluoromethoxy indanones were accessed, depended on the use of cinchonidine and cinchonine-derived catalyst. The method was extended to the enantioselective allylation reaction of $\alpha$-trifluoromethoxy indanones to give the allylation products in moderate yield with good enantioselectivity (up to $76 \%$ ee).
\end{abstract}

Keywords: trifluoromethoxy; fluorine; enantioselective; phase-transfer catalyst; organo-catalysis

\section{Introduction}

The role of fluorine in medicinal chemistry is expanding rapidly after it was discovered that the introduction of fluorine into an organic molecule could productively influence its physical and chemical properties [1-14]. In particular, the trifluoromethoxy $\left(\mathrm{OCF}_{3}\right)$ functional group has received extensive attention in recent years in the fields of pharmaceuticals and agrochemicals, owing to its unique three-dimensional electronic properties, suitable lipophilic properties, and good metabolic stability [15-20]. In fact, the $\mathrm{OCF}_{3}$ group is present in more than 1393 biologically active organic compounds according to a check of the PubChem database in June 2019 [21-23]. Compared to trifluoromethyl $\left(\mathrm{CF}_{3} ; \pi \mathrm{x}=0.88\right)$, methyl $\left(\mathrm{CH}_{3} ; \pi \mathrm{x}=0.52\right)$ and methoxy $\left(\mathrm{OCH}_{3}\right)$ groups $(\pi \mathrm{x}=-0.02)$, the $\mathrm{OCF}_{3}$ group has the highest lipophilicity value $(\pi \mathrm{x}=1.04)$ [24-29] resulting in the potential improvement of metabolic profiles, including permeability and absorption, when it is introduced into the appropriate position of parent molecules.

In contrast to the requirement of $\mathrm{OCF}_{3}$-containing drug candidates in medicinal chemistry, the synthesis of $\mathrm{OCF}_{3}$-containing organic compounds is relatively problematic. The $\mathrm{OCF}_{3}$ unit is traditionally synthesized from its chlorinated precursor, the trichloromethoxy $\left(\mathrm{OCCl}_{3}\right)$ moiety, by a chlorine/fluorine exchange reaction under harsh reaction conditions [30-35]. The $\mathrm{OCF}_{3}$ anion is unstable and decomposes rapidly into difluorophosgene $\left(\mathrm{O}=\mathrm{CF}_{2}\right)$ and a fluoride anion $\left(\mathrm{F}^{-}\right)$, which can make nucleophilic trifluoromethylation difficult [36]. The electrophilic trifluoromethylation of hydroxyl compounds is another strategy, but the method is somewhat limited. While the synthesis of $\mathrm{OCF}_{3}$-containing organic compounds has improved dramatically over the last five years [37-42], a method that can be used to construct a chiral " $\mathrm{C}^{*}-\mathrm{OCF}_{3}$ " unit is still extremely 
scarce. This should be one of the reasons of no pharmaceuticals with chiral, aliphatic " $\mathrm{C}^{*}-\mathrm{OCF}_{3}$ " unit reported. In 2017, Tang and co-workers reported the enantioselective bromo-trifluoromethoxylation of olefins by trifluoromethyl arylsulfonate (TFMS) under silver catalysis in the presence of 1,3-dibromo-5,5-dimethylhydantoin (DBDMH; Scheme 1a) [43]. Later, Shen and co-workers reported a method to construct chiral trifluoromethoxyl compounds by the Ni-catalyzed enantioselective Suzuki-Miyaura coupling of secondary benzyl bromides in good to high enantioselectivity (Scheme 1b) [44]. We developed a strategy for the synthesis of chiral, non-racemic $\alpha-\mathrm{OCF}_{3}-$ ketones with a tetrasubstituted carbon center via a Pd-catalyzed enantioselective Tsuji-allylation reaction with high enantioselectivity (Scheme 1c) $[45,46]$. Very recently, Liu and co-workers reported the Pd-catalyzed enantioselective intramolecular trifluoromethoxylation reaction of alkenes using $\mathrm{CsOCF}_{3}$ to furnish $\mathrm{OCF}_{3}$-compounds with a chiral stereogenic center (Scheme 1d) [47]. While these methods have broad substrate scopes with high enantioselectivity, all the methods require transition metal catalysts. Herein, we report the first example of constructing molecules with an $\mathrm{OCF}_{3}$ chiral center under non-metallic, organocatalytic conditions. The $\alpha-\mathrm{OCF}_{3}$ indanones react with benzyl bromides in the presence of a cinchona alkaloid-derived chiral phase-transfer catalyst (PTC) to afford enantioenriched $\alpha$-benzyl- $\alpha-\mathrm{OCF}_{3}$ indanones in high yield with up to $57 \%$ ee. Access to both $(R)$ - and (S)-enantiomers of $\alpha$-benzyl- $\alpha-\mathrm{OCF}_{3}$ indanones can be controlled by the catalysts. The method was expanded to the enantioselective allylation reaction with allyl bromide to provide $\alpha$-allyl- $\alpha-\mathrm{OCF}_{3}$ indanones with up to $76 \%$ ee (Scheme 1e).

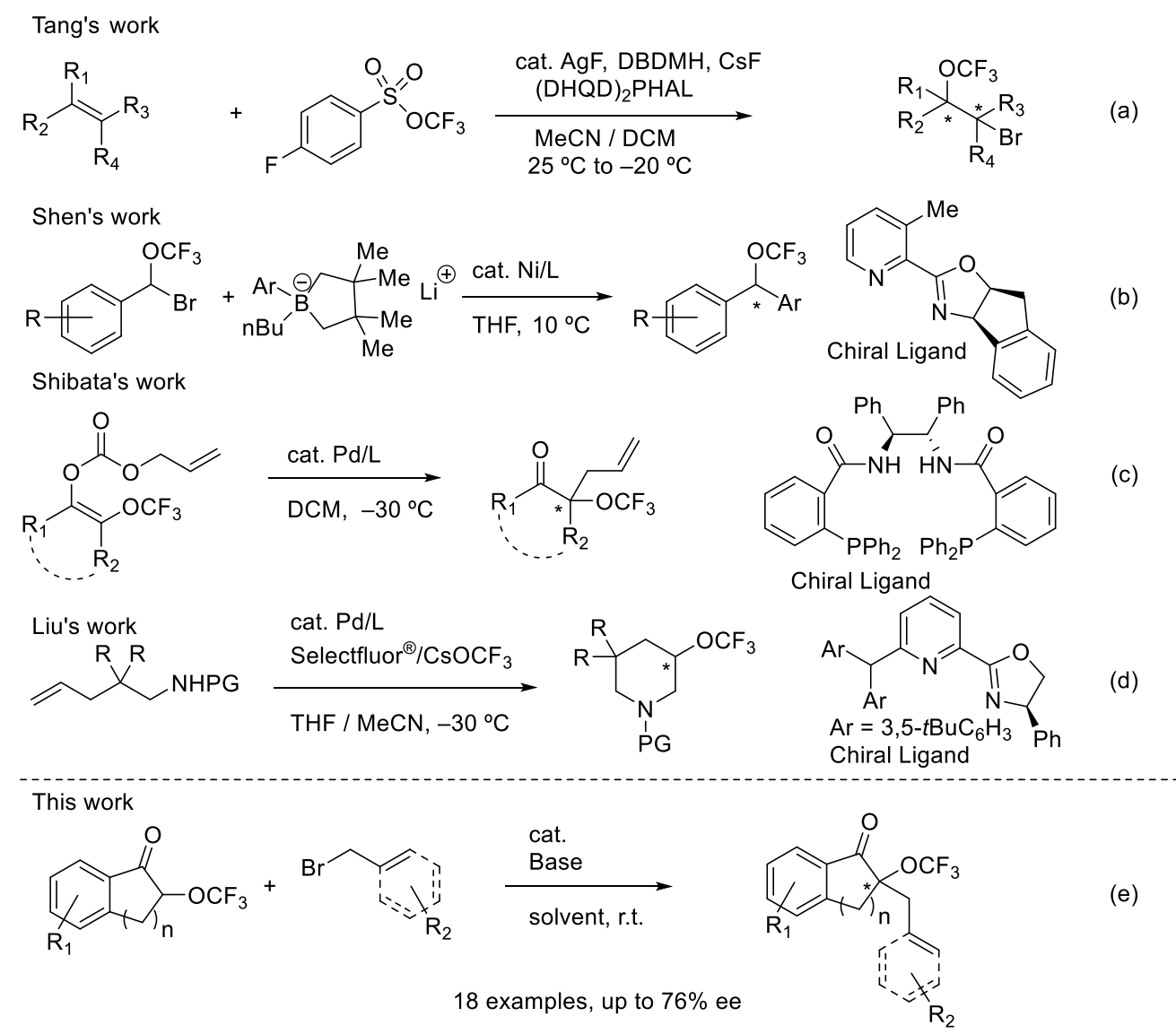

Scheme 1. Enantioselective synthesis of trifluoromethoxy-containing compounds.

\section{Results and Discussion}

The enantioselective benzylation of $\alpha-\mathrm{OCF}_{3}$-substituted indanone 1a with benzyl bromide (2a) was first examined (Table 1). The screening of representative cinchonine-derived PTCs, CN-1-CN-8 (entries 1-8) in toluene revealed that $\mathrm{N}$-[4-(trifluoromethyl)benzyl]cinchoninium bromide $\mathrm{CN}-4$ 
exhibited potential performance with $88 \%$ yield with $26 \%$ ee of (+)-3aa. We next examined solvents (n-hexane, dichloromethane (DCM), tetrahydrofuran (THF), diethyl ether $\left(\mathrm{Et}_{2} \mathrm{O}\right)$ and $\mathrm{N}, \mathrm{N}$-dimethylformamide (DMF)), but no satisfying consequences were observed (entries 9-13). Bases were next examined (potassium carbonate $\left(\mathrm{K}_{2} \mathrm{CO}_{3}\right)$, sodium hydroxide $(\mathrm{NaOH})$, cesium hydroxide monohydrate $\left(\mathrm{CsOH} \cdot \mathrm{H}_{2} \mathrm{O}\right)$, lithium hydroxide $(\mathrm{LiOH})$, sodium hydride $(\mathrm{NaH})$, potassium acetate $(\mathrm{KOAc})$, dipotassium phosphate $\left(\mathrm{K}_{2} \mathrm{HPO}_{4}\right)$ potassium tert-butoxide $(\mathrm{KO} \mathrm{BBu})$ and potassium methoxide (KOMe; entries 14-22). A 50\% $\mathrm{KOH}$ aqueous solution and the addition 2 equiv water with $\mathrm{KOH}$ were also tested (entries 23-24), the results showed $\mathrm{H}_{2} \mathrm{O}$ could not increase yield or enantioselectivity. Among these, $\mathrm{CsOH} \cdot \mathrm{H}_{2} \mathrm{O}$ exhibited the best performance ( $83 \%$ yield) with $43 \%$ ee of (+)-3aa. Additional solvent screening using $\mathrm{CsOH} \cdot \mathrm{H}_{2} \mathrm{O}$ (benzotrifluoride $\left(\mathrm{PhCF}_{3}\right)$, toluene: $\mathrm{CHCl}_{3}=7: 3$; entries 25-26) revealed no improvement in the results. Additional catalyst CN-9 was used, racemic product was obtained (entry 27). This result indicated that a free-OH group on the phase-transfer catalyst should play an important role for the induction of enantioselectivity, such as hydrogen bonding. The concentration of the reaction $(0.1 \mathrm{M}$ to $0.02 \mathrm{M})$ and the temperature affected selectivity (entries 28-29), and the best results obtained were $80 \%$ yield with $54 \%$ ee (entry 30 ). The product with an opposite configuration, (-)-3aa was obtained in $75 \%$ yield with $50 \%$ ee using CD-4 (entry 32). More optimization results using other PTC were shown in the supporting information (SI; Table S1, in SI).<smiles>O=C1c2ccccc2CC1OC(F)(F)F</smiles>

1a

Table 1. Optimal condition screening ${ }^{1}$.
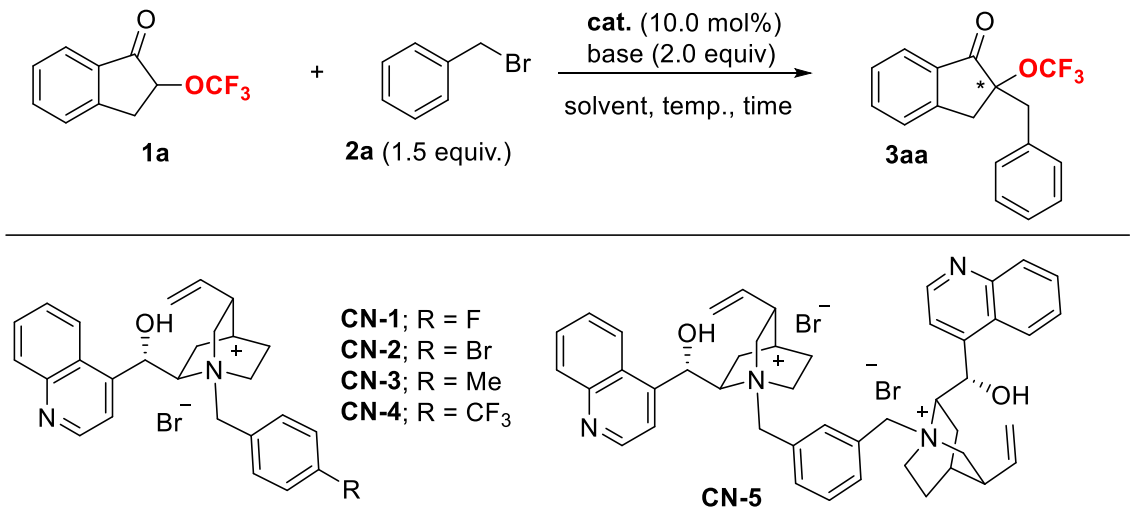<smiles>O=CC1CC2CCC1C2[C@H](O)c1ccnc2ccccc12</smiles>

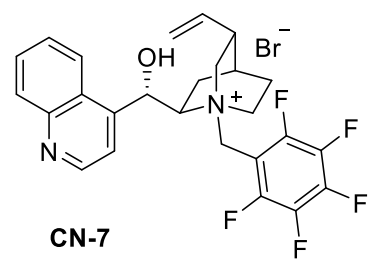

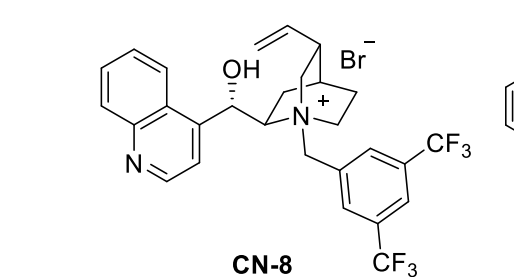<smiles>C=CCOC(C[N+]1(Cc2ccc(C(F)(F)F)cc2)CCC1C(C=C)CC)c1ccnc2ccccc12</smiles><smiles>C=CC1CC2CCC1C[N+]2(Cc1ccc(C(F)(F)F)cc1)CC(O)c1ccnc2ccccc12</smiles>

\begin{tabular}{ccccccc}
\hline Entry & Cat. & Base & Solvent & Time & Yield (\%) $^{\mathbf{7}}$ & ee (\%) $^{\mathbf{8}}$ \\
\hline 1 & $\mathrm{CN}-1$ & $\mathrm{KOH}$ & Toluene & 15 & 73 & 1 \\
2 & $\mathrm{CN}-2$ & $\mathrm{KOH}$ & Toluene & 15 & 75 & 3 \\
3 & $\mathrm{CN}-3$ & $\mathrm{KOH}$ & Toluene & 15 & 82 & 14 \\
\hline
\end{tabular}


Table 1. Cont.

\begin{tabular}{|c|c|c|c|c|c|c|}
\hline Entry & Cat. & Base & Solvent & Time & Yield $(\%)^{7}$ & ee $(\%)^{8}$ \\
\hline 4 & $\mathrm{CN}-4$ & $\mathrm{KOH}$ & Toluene & 15 & 88 & 26 \\
\hline 5 & CN-5 & $\mathrm{KOH}$ & Toluene & 15 & 82 & 14 \\
\hline 6 & CN-6 & $\mathrm{KOH}$ & Toluene & 15 & 89 & 0 \\
\hline 7 & $\mathrm{CN}-7$ & $\mathrm{KOH}$ & Toluene & 15 & 67 & 0 \\
\hline 8 & CN-8 & $\mathrm{KOH}$ & Toluene & 15 & 78 & 0 \\
\hline 9 & CN-4 & $\mathrm{KOH}$ & $n$-hexane & 24 & 38 & 2 \\
\hline 10 & CN-4 & $\mathrm{KOH}$ & DCM & 24 & 65 & 5 \\
\hline 11 & CN-4 & $\mathrm{KOH}$ & THF & 24 & 68 & 5 \\
\hline 12 & $\mathrm{CN}-4$ & $\mathrm{KOH}$ & $\mathrm{Et}_{2} \mathrm{O}$ & 24 & 40 & 19 \\
\hline 13 & $\mathrm{CN}-4$ & $\mathrm{KOH}$ & DMF & 15 & 20 & 1 \\
\hline 14 & CN-4 & $\mathrm{K}_{2} \mathrm{CO}_{3}$ & Toluene & 48 & 11 & n.d. \\
\hline 15 & $\mathrm{CN}-4$ & $\mathrm{NaOH}$ & Toluene & 48 & 58 & 4 \\
\hline 16 & $\mathrm{CN}-4$ & $\mathrm{CsOH} \cdot \mathrm{H}_{2} \mathrm{O}$ & Toluene & 15 & 83 & 43 \\
\hline 17 & CN-4 & $\mathrm{LiOH}^{2}$ & Toluene & 48 & NR & - \\
\hline 18 & $\mathrm{CN}-4$ & $\mathrm{NaH}$ & Toluene & 48 & 57 & 0 \\
\hline 19 & CN-4 & KOAC & Toluene & 48 & NR & - \\
\hline 20 & $\mathrm{CN}-4$ & $\mathrm{~K}_{2} \mathrm{HPO}_{4}$ & Toluene & 48 & NR & - \\
\hline 21 & CN-4 & $\mathrm{KO} t \mathrm{Bu}$ & Toluene & 24 & 26 & 17 \\
\hline 22 & $\mathrm{CN}-4$ & KOMe & Toluene & 24 & 6 & n.d. \\
\hline 23 & CN-4 & $50 \% \mathrm{KOH}(\mathrm{aq})$ & Toluene & 15 & 44 & 6 \\
\hline $24^{2}$ & $\mathrm{CN}-4$ & $\mathrm{KOH}$ & Toluene & 15 & 68 & 24 \\
\hline 25 & $\mathrm{CN}-4$ & $\mathrm{CsOH} \cdot \mathrm{H}_{2} \mathrm{O}$ & $\mathrm{PhCF}_{3}$ & 15 & 96 & 9 \\
\hline 26 & $\mathrm{CN}-4$ & $\mathrm{CsOH} \cdot \mathrm{H}_{2} \mathrm{O}$ & Toluene: $\mathrm{CHCl}_{3}=7: 3$ & 24 & 39 & 35 \\
\hline 27 & CN-9 & $\mathrm{CsOH} \cdot \mathrm{H}_{2} \mathrm{O}$ & Toluene & 15 & 77 & 1 \\
\hline $28^{3}$ & $\mathrm{CN}-4$ & $\mathrm{CsOH} \cdot \mathrm{H}_{2} \mathrm{O}$ & Toluene & 15 & 92 & 49 \\
\hline $29^{4}$ & $\mathrm{CN}-4$ & $\mathrm{CsOH} \cdot \mathrm{H}_{2} \mathrm{O}$ & Toluene & 15 & 83 & 50 \\
\hline $30^{5}$ & $\mathrm{CN}-4$ & $\mathrm{CsOH} \cdot \mathrm{H}_{2} \mathrm{O}$ & Toluene & 15 & 80 & 54 \\
\hline $31^{5,6}$ & $\mathrm{CN}-4$ & $\mathrm{CsOH} \cdot \mathrm{H}_{2} \mathrm{O}$ & Toluene & 72 & 75 & 57 \\
\hline $32^{5}$ & CD-4 & $\mathrm{CsOH} \cdot \mathrm{H}_{2} \mathrm{O}$ & Toluene & 15 & 75 & -50 \\
\hline
\end{tabular}

1 Reaction conditions: 1a (0.1 mmol, 1.0 equiv), BnBr 2a (0.15 mmol 1.5 equiv), base (0.2 mmol, 2.0 equiv) and cat. (10.0 mol\%) were stirred in $1.0 \mathrm{~mL}$ of anhydrous toluene at room temperature. ${ }^{2} \mathrm{H}_{2} \mathrm{O}$ (2.0 equiv) was added. ${ }^{3} 2.0 \mathrm{~mL}$ of toluene was used. ${ }^{4} 3.0 \mathrm{~mL}$ of toluene was used. ${ }^{5} 5.0 \mathrm{~mL}$ of toluene was used. ${ }^{6}$ Stirred at $0{ }^{\circ} \mathrm{C} .{ }^{7}$ Isolated yields. ${ }^{8}$ ee was determined by chiral HPLC. $\mathrm{CN}=$ cinchonine, $\mathrm{CD}=$ cinchonidine.

With the optimal reaction conditions in hand (Table 1, entry 30), we explored the substrate scope of this enantioselective catalytic benzylation of $\alpha-\mathrm{OCF}_{3}$ indanones 1 (Scheme 2). With a variety of benzyl bromides $\mathbf{2} \mathbf{b}-\mathbf{2} \mathbf{g}$ under the optimal conditions, the desired $\mathrm{OCF}_{3}$ indanones (+)-3ab-3ah were obtained in good to high yield $(63 \%-85 \%)$ with moderate ee $(24 \%-53 \%)$. Indanones with an electron-withdrawing group on the aromatic ring (1b-1d) gave the desired products (+)-3ba-3da in high to excellent yield $(73 \%-93 \%)$ with good ee $(51 \%-57 \%)$. On the other hand, indanones with an electron-donating group (1e-1g) furnished products (+)-3ea-3ga in high to excellent yield, but the ee decreased (13\%-33\% ee). The method was unsuitable for the benzylation of $\alpha-\mathrm{OCF}_{3}$ tetralone $1 \mathrm{~h}$ under the same conditions; the corresponding product 3 ha was detected in $51 \%$ yield with $8 \%$ ee.

The substrate scope of the enantioselective benzylation of $\alpha-\mathrm{OCF}_{3}$ indanones 1 using a catalyst, CN-4, under the same reaction conditions furnished (-)-3 ((S)-3) with an opposite configuration in similar yield and up to 50\% ee (Scheme 3). The absolute stereochemistry of the (+)-3 was temporality assigned to be $(R)$ based on the results for the enantioselective allylation of $\mathbf{1}$ with allyl bromide (2i) as discussed below (see the later part of this paper, Scheme 4). 


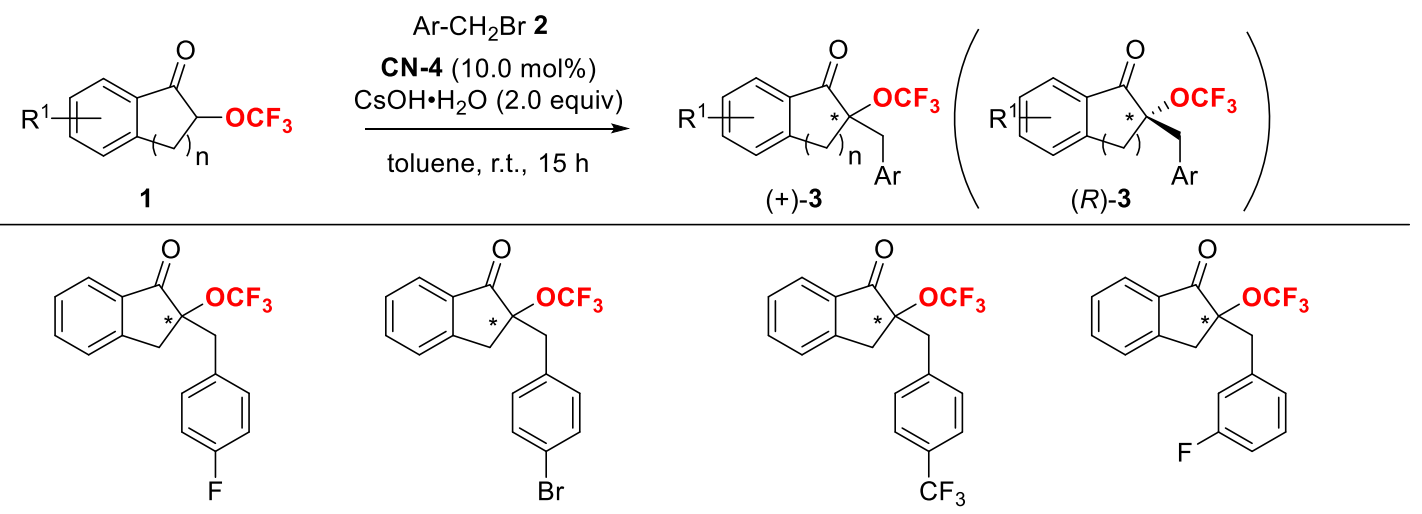

(+)-3ab, $83 \%$ yield, $53 \%$ ee (+)-3ac, $75 \%$ yield, $41 \%$ ee

(+)-3ad, $63 \%$ yield, $40 \%$ ee (+)-3ae, $75 \%$ yield, $47 \%$ ee<smiles>O=C1c2ccccc2CC1(Cc1ccc(-c2ccccc2)cc1)OC(F)(F)F</smiles><smiles>O=C1c2ccccc2CC1(Cc1ccc2ccccc2c1)OC(F)(F)F</smiles><smiles>CC(C)(C)c1cc(CC2(OC(F)(F)F)Cc3ccccc3C2=O)cc(C(C)(C)C)c1</smiles><smiles>O=C1c2ccc(Br)cc2CC1(Cc1ccccc1)OC(F)(F)F</smiles><smiles>O=C1c2ccc(F)cc2CC1(Cc1ccccc1)OC(F)(F)F</smiles>

(+)-3af, $81 \%$ yield

(+)-3ag, $85 \%$ yield, $41 \%$ ee $24 \%$ ee

(+)-3ah, $79 \%$ yield, $37 \%$ ee

(+)-3ba, $93 \%$ yield, $57 \%$ ee (+)-3ca, $71 \%$ yield $51 \%$ ee<smiles>COc1ccc2c(c1)C(=O)[C@](Cc1ccccc1)(OC(F)(F)F)C2</smiles>
(+)-3da, $82 \%$ yield,
(+)-3ea, $85 \%$ yield,
(+)-3fa, $92 \%$ yield,
(+)-3ga, 68\% yield,
(+)-3ha; $51 \%$ yield
$8 \%$ ee $56 \%$ ee

$29 \%$ ee

$33 \%$ ee

$13 \%$ ee

Scheme 2. The enantioselective benzylation of $\alpha$-trifluoromethoxy ketones 1 under $\mathrm{CN}-4$ catalysis.

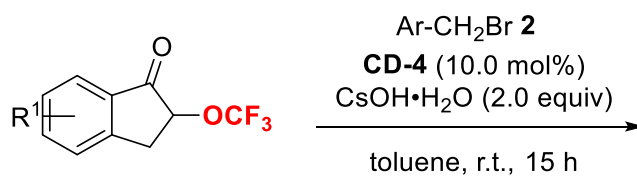

1<smiles>O=C1c2ccccc2CC1(Cc1ccccc1)OC(F)(F)F</smiles>

(-)-3aa, $62 \%$ yield, $50 \%$ ee<smiles>O=C1c2ccccc2CC1(Cc1ccc(-c2ccccc2)cc1)OC(F)(F)F</smiles>

$(-)-3 a b, 63 \%$ yield, $24 \%$ ee

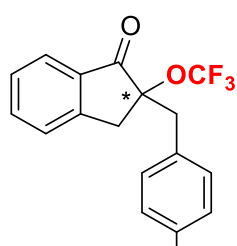

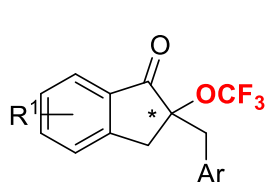

$(-)-3$

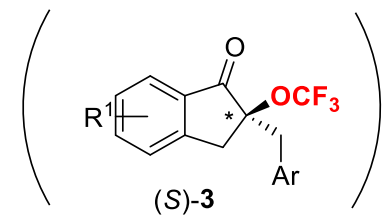

(S)-3

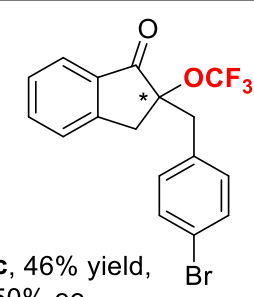
$50 \%$ ee

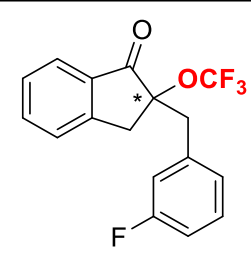

(-)-3ae, $51 \%$ yield,

(-)-3af, $60 \%$ yield, $23 \%$ ee

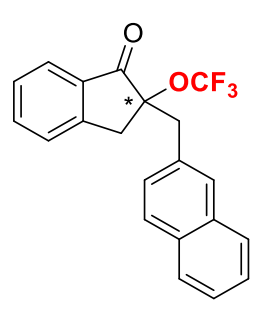

(-)-3ag, $70 \%$ yield, $31 \%$ ee

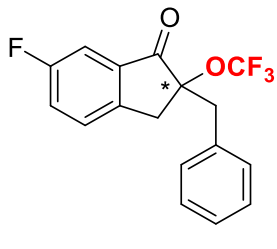

(-)-3da, $66 \%$ yield, $34 \%$ ee

Scheme 3. The enantioselective benzylation of $\alpha$-trifluoromethoxy indanones 1 under CD-4 catalysis. 
It should be noted that the method could be applied for the enantioselective allylation of $\alpha-\mathrm{OCF}_{3}$ indanones 1 with allyl bromide (2i) under $\mathrm{CN}-4$ or CD-4 catalysis. The desired (+)- and $(-)-\alpha$-allyl- $\alpha-\mathrm{OCF}_{3}$ indanones 3ai were obtained in moderate yield with up to $70 \%$ ee and $76 \%$ ee, respectively (Scheme 4$)$. The absolute configurations of 3ai were determined to be the $(R)$-configuration for (+)-3ai and the (S)-configuration for (-)-3ai by comparing to the optical rotation of reported $(S)$-3ai $\left([\alpha]_{\mathrm{D}}^{25}=-22.5\right)$ [45]. The higher enantioselectivity with allylic substrates is most likely due to the less steric hindrance than benzyl bromides.

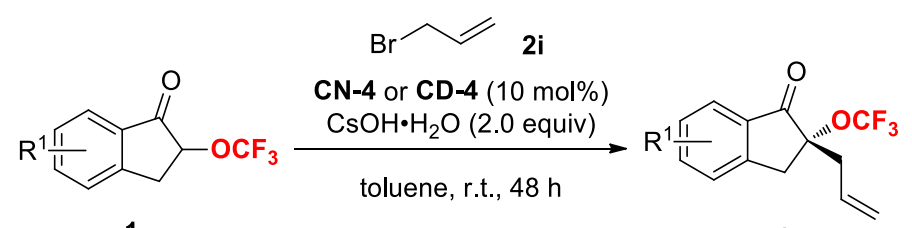

1

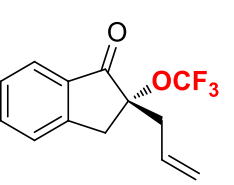

(R)-3ai, $43 \%$ yield, $68 \%$ ee, $[\alpha]^{25}=+16.5$<smiles>C=CC[C@]1(OC(F)(F)F)Cc2ccccc2C1=O</smiles>

(S)-3ai, $42 \%$ yield, $62 \%$ ee, $[\alpha]^{25}{ }_{D}=-15.1$ $81 \%$ ee,$[\alpha]_{D}^{25}=-22.5$ in Ref. [45]
$(R)-3$

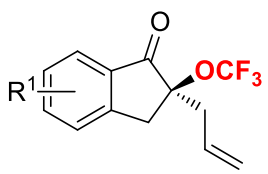

(S)-3

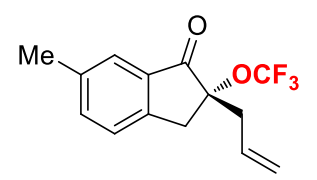

(R)-3ci, 35\% yield, $70 \%$ ee

(R)-3ei, $52 \%$ yield, $45 \%$ ee<smiles>C=CC[C@]1(OC(F)(F)F)Cc2cc(F)ccc2C1=O</smiles>

(S)-3ci, $31 \%$ yield, $76 \%$ ee

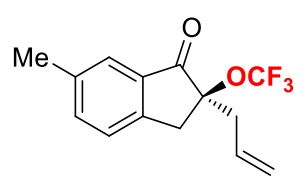

(S)-3ei, $43 \%$ yield, $62 \%$ ee

Scheme 4. The enantioselective allylation of $\alpha$-trifluoromethoxy indanones 1 under CN-4 or CD-4 catalysis. For (R)-3, CN-4 was used. For $(S)-3$, CD-4 was used.

\section{Materials and Methods}

\subsection{General Information}

All reagents were used as received from commercial sources, unless specified otherwise. All reactions were performed in oven-dried glassware under a positive pressure of nitrogen. Solvents were transferred via syringe and were introduced into the reaction vessels though a rubber septum. All reactions were monitored by thin-layer chromatography (TLC) carried out on 0.25 mm Merck silica-gel (60-F254) (Kenilworth, NJ, USA). The TLC plates were visualized with UV light (254 nm) (Tokyo, Japan) and $p$-Anisaldehyde in ethanol/heat. Column chromatography was carried out on a column packed with silica-gel $60 \mathrm{~N}$ spherical neutral size $63-210 \mu \mathrm{m}$ or $40-63 \mu \mathrm{m}$. The ${ }^{1} \mathrm{H}-\mathrm{NMR}$ (300 MHz), ${ }^{19} \mathrm{~F}-\mathrm{NMR}(282 \mathrm{MHz})$ and ${ }^{13} \mathrm{C}-\mathrm{NMR}(126 \mathrm{MHz})$ spectra for solution in $\mathrm{CDCl}_{3}$ were recorded on a Bruker Avance 500 (Karlsruhe, Germany), Varian Mercury 300 (Palo Alto, CA, USA). Chemical shifts $(\delta)$ are expressed in ppm downfield from tetramethylsilane $(\delta \mathrm{H}=0.00 \mathrm{ppm})$ or tetramethylsilane $(\delta \mathrm{C}=0.00 \mathrm{ppm})$ or hexafluorobenzene $(\delta \mathrm{F}=-162.20 \mathrm{ppm})$. Optical rotations were measured with a Horiba SEPA-300 operating at $589 \mathrm{~nm}$ (Kyoto, Japan). Mass spectra were recorded on an LCMS-2020EV (ESI-MS) system (Shimadzu Corporation, Kyoto, Japan). High resolution mass spectrometry (HRMS) was recorded on a Waters Synapt G2 HDMS (ESI-MS) (Milford, MA, USA). The wave numbers (v) of recorded IR-signals are quoted in $\mathrm{cm}^{-1}$ on a JASCO FT/IR-4100 spectrometer (Tokyo, Japan). HPLC analyses were performed on a JASCOLC-2000 Plus series (Tokyo, Japan) using $4.6 \times 250 \mathrm{~mm}$ CHIRALCEL ${ }^{\circledR}$ series or CHIRALPAK series (Tokyo, Japan). The melting point was recorded on a BUCHI M-565 (Flawil, Switzerland). All solvents were dried and distilled before use. The ${ }^{1} \mathrm{H}$, ${ }^{13} \mathrm{C}$ and ${ }^{19} \mathrm{~F}-\mathrm{NMR}$ spectra of compounds 3 and HPLC data of compounds 3 are available in the Supplementary Material. 


\subsection{Preparation of $\alpha-\mathrm{OCF}_{3}-$ Substituted Indanones (General Procedure)}

All the substrates, $\alpha-\mathrm{OCF}_{3}$-indanones $\mathbf{1}$, were prepared by following a reported procedure [45]. General Procedure:

A mixture of the indanone (1.0 equiv) and $\mathrm{KOH}$ (3.0 equiv) in $\mathrm{MeOH}(0.4 \mathrm{M}$ ) was stirred for $15 \mathrm{~min}$ at $0{ }^{\circ} \mathrm{C}$, and $\mathrm{PhI}(\mathrm{OAc})_{2}$ (1.1 equiv) was added in 4-5 portions during $5 \mathrm{~min}$. The mixture was stirred at the same temperature for $1 \mathrm{~h}$, then warmed to room temperature and stirred overnight. The mixture was concentrated, dissolved in $\mathrm{Et}_{2} \mathrm{O}$, washed with $\mathrm{NaHCO}_{3}$ aq., dried over $\mathrm{Na}_{2} \mathrm{SO}_{4}$ and concentrated, then purified by silica-gel column chromatography. The pure product was then dissolved in EtOH $(0.3 \mathrm{M})$, and $3 \mathrm{~N} \mathrm{HCl}$ aq. $(1.0 \mathrm{M})$ was added. After stirring for $0.5 \mathrm{~h}$ at room temperature, the resulting mixture was extracted with $\mathrm{Et}_{2} \mathrm{O}$, and the combined organic layer was washed with sat. $\mathrm{NaHCO}_{3}$ aq. and brine, then dried over $\mathrm{Na}_{2} \mathrm{SO}_{4}$. The residue can be used without further purification for the next reaction.

A flask was charged with hydroxyketone, AgOTf (3.0 equiv), KF (4.0 equiv) and Selectfluor ${ }^{\circledR}$ (1.5 equiv) in a nitrogen-filled glovebox. Then ethyl acetate ( $0.2 \mathrm{M}), 2$-fluoropyridine (3.0 equiv) and $\mathrm{Me}_{3} \mathrm{SiCF}_{3}$ (3.0 equiv) were added successively under an Ar atmosphere. The resulting mixture was stirred overnight at room temperature. The reaction mixture was filtered through a pad of silica-gel and concentrated. The residue was purified by flash silica-gel column chromatography.

2-(Trifluoromethoxy)-2,3-dihydro-1H-inden-1-one (1a). The reaction was run according to the general procedure, and the product was consistent with previously reported characterization data [45]. ${ }^{1} \mathrm{H}-\mathrm{NMR}$ $\left(300 \mathrm{MHz}_{1} \mathrm{CDCl}_{3}\right) \delta: 7.82(\mathrm{~d}, J=6.9 \mathrm{~Hz}, 1 \mathrm{H}), 7.71-7.66(\mathrm{~m}, 1 \mathrm{H}), 7.49-7.42(\mathrm{~m}, 2 \mathrm{H}), 4.98-4.91(\mathrm{~m}, 1 \mathrm{H})$, $3.68(\mathrm{dd}, J=17.0,7.9 \mathrm{~Hz}, 1 \mathrm{H})$ and $3.26(\mathrm{dd}, J=17.0,5.0 \mathrm{~Hz}, 1 \mathrm{H}) \mathrm{ppm} .{ }^{19} \mathrm{~F}-\mathrm{NMR}\left(282 \mathrm{MHz}, \mathrm{CDCl}_{3}\right) \delta$ : $-59.66(\mathrm{~s}, 3 \mathrm{~F}) \mathrm{ppm}$.

5-Bromo-2-(trifluoromethoxy)-2,3-dihydro-1H-inden-1-one (1) ). The reaction was run according to the general procedure, and the product was consistent with previously reported characterization data [45]. ${ }^{1} \mathrm{H}-\mathrm{NMR}\left(300 \mathrm{MHz}, \mathrm{CDCl}_{3}\right) \delta: 7.70-7.58(\mathrm{~m}, 3 \mathrm{H}), 4.94-4.90(\mathrm{~m}, 1 \mathrm{H}), 3.66(\mathrm{dd}, J=17.2,7.9 \mathrm{~Hz}, 1 \mathrm{H})$ and $3.25(\mathrm{dd}, J=17.2,4.9 \mathrm{~Hz}, 1 \mathrm{H})$ ppm. ${ }^{19} \mathrm{~F}-\mathrm{NMR}\left(282 \mathrm{MHz}, \mathrm{CDCl}_{3}\right) \delta:-59.82(\mathrm{~s}, 3 \mathrm{~F}) \mathrm{ppm}$.

5-Fluoro-2-(trifluoromethoxy)-2,3-dihydro-1H-inden-1-one (1c). The reaction was run according to the general procedure, and the product was consistent with previously reported characterization data [45]. ${ }^{1} \mathrm{H}-\mathrm{NMR}\left(300 \mathrm{MHz}, \mathrm{CDCl}_{3}\right) \delta: 7.85(\mathrm{dd}, J=9.2,5.4 \mathrm{~Hz}, 1 \mathrm{H}), 7.19-7.13(\mathrm{~m}, 2 \mathrm{H}), 4.94(\mathrm{dd}, J=7.9,4.8 \mathrm{~Hz}$, $1 \mathrm{H}), 3.67(\mathrm{dd}, J=17.3,7.9 \mathrm{~Hz}, 1 \mathrm{H})$ and $3.26(\mathrm{dd}, J=17.3,4.9 \mathrm{~Hz}, 1 \mathrm{H}) \mathrm{ppm} .{ }^{19} \mathrm{~F}-\mathrm{NMR}\left(282 \mathrm{MHz}, \mathrm{CDCl}_{3}\right)$ $\delta:-59.81(\mathrm{~s}, 3 \mathrm{~F})$ and $-99.41(\mathrm{q}, J=8.1 \mathrm{~Hz}, 1 \mathrm{~F}) \mathrm{ppm}$.

6-Fluoro-2-(trifluoromethoxy)-2,3-dihydro-1H-inden-1-one (1d). The reaction was run according to the general procedure. ${ }^{1} \mathrm{H}-\mathrm{NMR}\left(300 \mathrm{MHz}, \mathrm{CDCl}_{3}\right) \delta: 7.48-7.37(\mathrm{~m}, 3 \mathrm{H}), 4.96(\mathrm{dd}, J=8.0,4.8 \mathrm{~Hz}, 1 \mathrm{H}), 3.66$ $(\mathrm{dd}, J=16.8,7.9 \mathrm{~Hz}, 1 \mathrm{H}), 3.22(\mathrm{dd}, J=16.8,5.0 \mathrm{~Hz}, 1 \mathrm{H}) \mathrm{ppm} .{ }^{19} \mathrm{~F}-\mathrm{NMR}\left(282 \mathrm{MHz}, \mathrm{CDCl}_{3}\right) \delta:-59.85(\mathrm{~s}$, 3F), -111.30--112.37 (m, 1F) ppm. ${ }^{13} \mathrm{C}-\mathrm{NMR}\left(126 \mathrm{MHz}, \mathrm{CDCl}_{3}\right) \delta: 196.73(\mathrm{~d}, \mathrm{~J}=3.0 \mathrm{~Hz}), 162.72(\mathrm{~d}$, $J=250.2 \mathrm{~Hz}), 145.06(\mathrm{~d}, J=2.4 \mathrm{~Hz}), 135.29(\mathrm{~d}, J=7.7 \mathrm{~Hz}), 128.34(\mathrm{~d}, J=7.8 \mathrm{~Hz}), 124.25(\mathrm{~d}, J=23.8 \mathrm{~Hz})$, $121.88(\mathrm{q}, J=256.9 \mathrm{~Hz}), 110.70(\mathrm{~d}, J=22.3 \mathrm{~Hz}), 76.85(\mathrm{q}, J=2.5 \mathrm{~Hz})$ and $33.19 \mathrm{ppm}$. MS (ESI): $\mathrm{m} / z 233$ $[\mathrm{M}-\mathrm{H}]^{-}$. m.p.: $93.4-95.1^{\circ} \mathrm{C}$.

6-Methyl-2-(trifluoromethoxy)-2,3-dihydro-1H-inden-1-one (1e). The reaction was run according to the general procedure, and the product was consistent with previously reported characterization data [45]. ${ }^{1} \mathrm{H}-\mathrm{NMR}\left(300 \mathrm{MHz}, \mathrm{CDCl}_{3}\right) \delta: 7.62(\mathrm{~s}, 1 \mathrm{H}), 7.50(\mathrm{~d}, J=7.5,1 \mathrm{H}), 7.35(\mathrm{~d}, J=7.9,1 \mathrm{H}), 4.92(\mathrm{dd}, J=7.9 \mathrm{~Hz}$, $1 \mathrm{H}), 3.67(\mathrm{dd}, J=17.3,7.9 \mathrm{~Hz}, 1 \mathrm{H})$ and $3.26(\mathrm{dd}, J=17.3,4.9 \mathrm{~Hz}, 1 \mathrm{H}) \mathrm{ppm} .{ }^{19} \mathrm{~F}-\mathrm{NMR}\left(282 \mathrm{MHz}, \mathrm{CDCl}_{3}\right)$ $\delta:-59.72(\mathrm{~s}, 3 \mathrm{~F}) \mathrm{ppm}$.

6-Methoxy-2-(trifluoromethoxy)-2,3-dihydro-1H-inden-1-one (1f). The reaction was run according to the general procedure, and the product was consistent with previously reported characterization data [45]. ${ }^{1} \mathrm{H}-\mathrm{NMR}\left(300 \mathrm{MHz} \mathrm{CDCl}_{3}\right) \delta: 7.37(\mathrm{~d}, J=8.3,1 \mathrm{H}), 7.29-7.23(\mathrm{~m}, 2 \mathrm{H}), 4.97-4.92(\mathrm{~m}, 1 \mathrm{H}), 3.86(\mathrm{~s}, 3 \mathrm{H})$, 
$3.61(\mathrm{dd}, J=16.6,7.8 \mathrm{~Hz}, 1 \mathrm{H})$ and $3.18(\mathrm{dd}, J=16.6,4.7 \mathrm{~Hz}, 1 \mathrm{H}) \mathrm{ppm} .{ }^{19} \mathrm{~F}-\mathrm{NMR}\left(282 \mathrm{MHz}, \mathrm{CDCl}_{3}\right) \delta$ : $-59.73(\mathrm{~s}, 3 \mathrm{~F}) \mathrm{ppm}$.

5,6-Dimethoxy-2-(trifluoromethoxy)-2,3-dihydro-1H-inden-1-one (19). The reaction was run according to the general procedure. ${ }^{1} \mathrm{H}-\mathrm{NMR}\left(300 \mathrm{MHz}, \mathrm{CDCl}_{3}\right) \delta: 7.24(\mathrm{~d}, J=14.6,1 \mathrm{H}), 4.90(\mathrm{dd}, J=7.6,4.3 \mathrm{~Hz}$, $1 \mathrm{H}), 3.99(\mathrm{~s}, 3 \mathrm{H}), 3.92(\mathrm{~s}, 3 \mathrm{H}), 3.58(\mathrm{dd}, J=16.8,7.6 \mathrm{~Hz}, 1 \mathrm{H})$ and $3.17(\mathrm{dd}, J=16.8,4.3 \mathrm{~Hz}, 1 \mathrm{H}) \mathrm{ppm}$. ${ }^{19} \mathrm{~F}-\mathrm{NMR}\left(282 \mathrm{MHz}, \mathrm{CDCl}_{3}\right.$ ) $\delta:-59.61$ (s, 3F) ppm. ${ }^{13} \mathrm{C}-\mathrm{NMR}\left(126 \mathrm{MHz}, \mathrm{CDCl}_{3}\right)$ ): 195.90, 156.89, $150.23,145.38,126.50,121.97(\mathrm{q}, J=256.5 \mathrm{~Hz}), 107.30,104.91,76.51(\mathrm{q}, J=2.3 \mathrm{~Hz}), 56.44,56.22$ and 33.54 ppm. MS (ESI): $\mathrm{m} / \mathrm{z} 277[\mathrm{M}+\mathrm{H}]^{+}$. m.p.: $136.9-138.0^{\circ} \mathrm{C}$.

2-(Trifluoromethoxy)-3,4-dihydronaphthalen-1(2H)-one (1h). The reaction was run according to the general procedure, and the product was consistent with previously reported characterization data [45]. ${ }^{1} \mathrm{H}-\mathrm{NMR}$ $\left(300 \mathrm{MHz}, \mathrm{CDCl}_{3}\right) \delta: 8.07(\mathrm{~d}, J=7.9,1 \mathrm{H}), 7.54(\mathrm{t}, J=7.4,1 \mathrm{H}), 7.37(\mathrm{t}, J=7.7,1 \mathrm{H}), 4.85(\mathrm{dd}, J=12.1$, $4.8 \mathrm{~Hz}, 1 \mathrm{H}), 3.16(\mathrm{dd}, J=8.1,4.4 \mathrm{~Hz}, 2 \mathrm{H})$ and $2.57-2.36(\mathrm{~m}, 2 \mathrm{H}) \mathrm{ppm} .{ }^{19} \mathrm{~F}-\mathrm{NMR}\left(282 \mathrm{MHz}, \mathrm{CDCl}_{3}\right) \delta$ : $-59.05(\mathrm{~s}, 3 \mathrm{~F}) \mathrm{ppm}$.

\subsection{Representative Procedure for the Enantioselective Catalytic Phase Transfer Benzylation}

A flask was charged with $\alpha-\mathrm{OCF}_{3}$-indanone 1 ( $0.10 \mathrm{mmol}, 1.0$ equiv), $\mathrm{CsOH} \cdot \mathrm{H}_{2} \mathrm{O}(0.20 \mathrm{mmol}$, 2.0 equiv) and cat. $4(0.010 \mathrm{mmol}, 10.0 \mathrm{~mol} \%)$ in a nitrogen-filled glovebox. Then anhydrous toluene $(5.0 \mathrm{~mL}, 0.02 \mathrm{M})$ and 2 ( $0.15 \mathrm{mmol}, 1.5$ equiv) was added under an Ar atmosphere. The resulting mixture was stirred overnight or $48 \mathrm{~h}$ at room temperature. After that, the solvent was removed under reduced pressure and the residue was purified by flash silica-gel column chromatography.

(+)-2-Benzyl-2-(trifluoromethoxy)-2,3-dihydro-1H-inden-1-one ((+)-3aa). The reaction was run according to the general procedure. Eluent ( $n$-hexane/ethyl acetate $=15 / 1$ ). Slightly yellow oil; $24.5 \mathrm{mg} ; 80 \%$ yield. The enantiomeric excess ( $54 \%$ ee) was determined by chiral HPLC using CHIRALCEL ${ }^{\circledR}$ OJ-H column ( $n$-hexane/isopropanol $=99.0 / 1.0$, flow rate $1.0 \mathrm{~mL} / \mathrm{min}, \lambda=254 \mathrm{~nm}) \mathrm{t}$ (major) $=16.525 \mathrm{~min}, \mathrm{t}$ (minor) $=$ $11.367 \mathrm{~min}) .[\alpha]_{\mathrm{D}}^{25}=+37.7\left(\mathrm{CH}_{2} \mathrm{Cl}_{2}, c=0.62\right){ }^{1} \mathrm{H}-\mathrm{NMR}\left(300 \mathrm{MHz}, \mathrm{CDCl}_{3}\right) \delta: 7.78(\mathrm{~d}, J=7.7 \mathrm{~Hz}, 1 \mathrm{H}), 7.59$ $(\mathrm{t}, J=7.4 \mathrm{~Hz}, 1 \mathrm{H}), 7.40-7.32(\mathrm{~m}, 2 \mathrm{H}), 7.22-7.17(\mathrm{~m}, 5 \mathrm{H}), 3.43(\mathrm{~s}, 2 \mathrm{H}), 3.31(\mathrm{~d}, J=13.8 \mathrm{~Hz}, 1 \mathrm{H})$ and $3.01(\mathrm{~d}$, $J=13.8 \mathrm{~Hz}, 1 \mathrm{H}) \mathrm{ppm} .{ }^{13} \mathrm{C}-\mathrm{NMR}\left(126 \mathrm{MHz}, \mathrm{CDCl}_{3}\right) \delta: 200.07,149.53,136.16,133.82,133.42,130.51$, $128.31,128.25,127.38,126.27,124.97,121.14(\mathrm{q}, J=258.8 \mathrm{~Hz}), 87.04,42.22$ and $35.52 \mathrm{ppm} .{ }^{19} \mathrm{~F}-\mathrm{NMR}(282$ $\mathrm{MHz}, \mathrm{CDCl}_{3}$ ) $\delta:-51.67$ (s, 3F) ppm. IR (NaCl): $v=3033,2929,1730,1608,1496,1456,1265,1043,757$ and $701 \mathrm{~cm}^{-1}$. HRMS (ESI) $\mathrm{m} / z$ : $[\mathrm{M}+\mathrm{Na}]^{+}$Calcd. for $\mathrm{C}_{17} \mathrm{H}_{13} \mathrm{~F}_{3} \mathrm{NaO}_{2}{ }^{+}$329.0760; found 329.0765.

(+)-2-(4-Fluorobenzyl)-2-(trifluoromethoxy)-2,3-dihydro-1H-inden-1-one ((+)-3ab). The reaction was run according to the general procedure. Eluent $(n$-hexane/ethyl acetate $=15 / 1)$. Slightly yellow oil; $26.9 \mathrm{mg}$; $83 \%$ yield. The enantiomeric excess $\left(53 \%\right.$ ee) was determined by chiral HPLC using CHIRALCEL ${ }^{\circledR}$ OJ-H ( $n$-hexane/isopropanol $=99.0 / 1.0$, flow rate $1.0 \mathrm{~mL} / \mathrm{min}, \lambda=254 \mathrm{~nm}) \mathrm{t}($ major $)=19.383 \mathrm{~min}, \mathrm{t}$ (minor) $=11.833 \mathrm{~min}) .[\alpha]_{\mathrm{D}}^{25}=+29.6\left(\mathrm{CH}_{2} \mathrm{Cl}_{2}, c=0.76\right) .{ }^{1} \mathrm{H}-\mathrm{NMR}\left(300 \mathrm{MHz}, \mathrm{CDCl}_{3}\right) \delta: 7.77(\mathrm{~d}, J=7.7$ $\mathrm{Hz}, 1 \mathrm{H}), 7.61(\mathrm{t}, J=7.5 \mathrm{~Hz}, 1 \mathrm{H}), 7.41-7.33(\mathrm{~m}, 2 \mathrm{H}), 7.17-7.12(\mathrm{~m}, 2 \mathrm{H}), 6.91(\mathrm{t}, J=8.4 \mathrm{~Hz}, 2 \mathrm{H}), 3.48-3.35$ $(\mathrm{m}, 2 \mathrm{H}), 3.27(\mathrm{~d}, J=14.0 \mathrm{~Hz}, 1 \mathrm{H})$ and $3.00(\mathrm{~d}, J=14.0 \mathrm{~Hz}, 1 \mathrm{H}) \mathrm{ppm} .{ }^{13} \mathrm{C}-\mathrm{NMR}\left(126 \mathrm{MHz}, \mathrm{CDCl}_{3}\right) \delta$ : $199.84,162.16(\mathrm{~d}, J=246.2 \mathrm{~Hz}), 149.37,136.30,133.36,132.02(\mathrm{~d}, J=8.0 \mathrm{~Hz}), 129.50(\mathrm{~d}, J=3.2 \mathrm{~Hz}), 128.38$, $126.29,124.98,121.12(\mathrm{q}, J=258.8 \mathrm{~Hz}), 115.24(\mathrm{~d}, J=21.4 \mathrm{~Hz}), 86.87,41.50$ and $35.56 \mathrm{ppm} .{ }^{19} \mathrm{~F}-\mathrm{NMR}$ $\left(282 \mathrm{MHz}, \mathrm{CDCl}_{3}\right) \delta:-51.72(\mathrm{~s}, 3 \mathrm{~F})$ and $-115.49-115.59(\mathrm{~m}, 1 \mathrm{~F}) \mathrm{ppm}$. IR $(\mathrm{NaCl}): v=3045,2931,1730$, $1606,1512,1469,1265,1159,838$ and $744 \mathrm{~cm}^{-1}$. HRMS (ESI) $\mathrm{m} / \mathrm{z}$ : $[\mathrm{M}+\mathrm{Na}]^{+}$Calcd. for $\mathrm{C}_{17} \mathrm{H}_{12} \mathrm{~F}_{4} \mathrm{NaO}_{2}{ }^{+}$ 347.0666; found 347.0669.

(+)-2-(4-Bromobenzyl)-2-(trifluoromethoxy)-2,3-dihydro-1H-inden-1-one ((+)-3ac). The reaction was run according to the general procedure. Eluent ( $n$-hexane/ethyl acetate $=15 / 1)$. Slightly yellow semi-solid; $28.9 \mathrm{mg} ; 75 \%$ yield. The enantiomeric excess $(41 \%$ ee) was determined by chiral HPLC using CHIRALCEL ${ }^{\circledR}$ OJ-H (n-hexane/isopropanol $=99.0 / 1.0$, flow rate $\left.1.0 \mathrm{~mL} / \mathrm{min}, \lambda=254 \mathrm{~nm}\right) \mathrm{t}$ (major) = $33.433 \mathrm{~min}, \mathrm{t}$ (minor) $=13.825 \mathrm{~min}) .[\alpha]_{\mathrm{D}}^{25}=+22.8\left(\mathrm{CH}_{2} \mathrm{Cl}_{2}, c=0.84\right) .{ }^{1} \mathrm{H}-\mathrm{NMR}\left(300 \mathrm{MHz}, \mathrm{CDCl}_{3}\right) \delta$ : $7.79(\mathrm{~d}, J=7.7 \mathrm{~Hz}, 1 \mathrm{H}), 7.62(\mathrm{t}, J=7.5 \mathrm{~Hz}, 1 \mathrm{H}), 7.43-7.34(\mathrm{~m}, 4 \mathrm{H}), 7.07(\mathrm{~d}, J=8.2,2 \mathrm{H}), 3.47-3.33(\mathrm{~m}, 2 \mathrm{H})$, 
$3.25(\mathrm{~d}, J=14.0 \mathrm{~Hz}, 1 \mathrm{H})$ and $2.95(\mathrm{~d}, J=14.0 \mathrm{~Hz}, 1 \mathrm{H}) \mathrm{ppm} .{ }^{13} \mathrm{C}-\mathrm{NMR}\left(126 \mathrm{MHz}, \mathrm{CDCl}_{3}\right) \delta: 199.66$, $149.29,136.37,133.22,132.86,132.16,131.47,128.45,126.35,125.07,121.61,121.08$ (q, J = 259.0 Hz), 86.63, 41.65 and $35.54 \mathrm{ppm} .{ }^{19} \mathrm{~F}-\mathrm{NMR}\left(282 \mathrm{MHz}, \mathrm{CDCl}_{3}\right) \delta:-51.75(\mathrm{~s}, 3 \mathrm{~F}) \mathrm{ppm}$. IR (NaCl): $v=3074$, 2929, 1730, 1608, 1489, 1265, 1201, 1153, 1012 and $519 \mathrm{~cm}^{-1}$. HRMS (ESI) $\mathrm{m} / z:[\mathrm{M}+\mathrm{Na}]^{+}$Calcd. for $\mathrm{C}_{17} \mathrm{H}_{12} \mathrm{BrF}_{3} \mathrm{NaO}_{2}{ }^{+}$406.9865; found 406.9865 .

(+)-2-(Trifluoromethoxy)-2-(4-(trifluoromethyl)benzyl)-2,3-dihydro-1H-inden-1-one ((+)-3ad). The reaction was run according to the general procedure. Eluent $(n$-hexane/ethyl acetate $=15 / 1)$. Slightly yellow oil; $23.6 \mathrm{mg} ; 63 \%$ yield. The enantiomeric excess ( $40 \%$ ee) was determined by chiral HPLC using CHIRALCEL ${ }^{\circledR}$ OJ-H ( $n$-hexane/isopropanol $=99.0 / 1.0$, flow rate $\left.1.0 \mathrm{~mL} / \mathrm{min}, \lambda=254 \mathrm{~nm}\right) \mathrm{t}$ (major) $=$ 48.208 min, $\mathrm{t}$ (minor) $=33.525 \mathrm{~min}) .[\alpha]_{\mathrm{D}}^{25}=+18.8\left(\mathrm{CH}_{2} \mathrm{Cl}_{2}, c=0.50\right) .{ }^{1} \mathrm{H}-\mathrm{NMR}\left(300 \mathrm{MHz}, \mathrm{CDCl}_{3}\right) \delta$ : $7.80(\mathrm{~d}, J=7.7 \mathrm{~Hz}, 1 \mathrm{H}), 7.63(\mathrm{t}, J=7.5,1 \mathrm{H}), 7.51(\mathrm{~d}, J=8.0 \mathrm{~Hz}, 2 \mathrm{H}), 7.42(\mathrm{t}, J=7.4 \mathrm{~Hz}, 1 \mathrm{H}), 7.39-7.32(\mathrm{~m}$, $3 \mathrm{H}), 3.49-3.34(\mathrm{~m}, 3 \mathrm{H})$ and $3.04(\mathrm{~d}, J=13.9 \mathrm{~Hz}, 1 \mathrm{H}) \mathrm{ppm} .{ }^{13} \mathrm{C}-\mathrm{NMR}\left(126 \mathrm{MHz}, \mathrm{CDCl}_{3}\right) \delta: 199.42,149.15$, $138.06,136.45,133.13,130.87,129.72(\mathrm{q}, J=32.5 \mathrm{~Hz}), 128.54,126.39,125.25(\mathrm{q}, J=3.7 \mathrm{~Hz}), 125.15,124.00$ $(\mathrm{q}, J=272.1 \mathrm{~Hz}), 121.07(\mathrm{q}, J=259.2 \mathrm{~Hz}), 86.57,42.03$ and $35.65 \mathrm{ppm} .{ }^{19} \mathrm{~F}-\mathrm{NMR}\left(282 \mathrm{MHz}, \mathrm{CDCl}_{3}\right) \delta$ : $-51.82(\mathrm{~s}, 3 \mathrm{~F})$ and $-63.14(\mathrm{~s}, 3 \mathrm{~F}) \mathrm{ppm}$. IR $(\mathrm{NaCl}): v=3076,2937,1732,1610,1419,1327,1267,1162,1068$ and $748 \mathrm{~cm}^{-1}$. HRMS (ESI) $\mathrm{m} / z$ : $[\mathrm{M}+\mathrm{Na}]^{+}$Calcd. for $\mathrm{C}_{18} \mathrm{H}_{12} \mathrm{~F}_{6} \mathrm{NaO}_{2}{ }^{+}$397.0634; found 397.0638.

(+)-2-(3-Fluorobenzyl)-2-(trifluoromethoxy)-2,3-dihydro-1H-inden-1-one ((+)-3ae). The reaction was run according to the general procedure. Eluent $(n$-hexane/ethyl acetate $=15 / 1)$. Slightly yellow oil; $24.3 \mathrm{mg}$; $75 \%$ yield. The enantiomeric excess $\left(47 \%\right.$ ee) was determined by chiral HPLC using CHIRALCEL ${ }^{\circledR}$ OJ-H ( $n$-hexane/isopropanol $=99.0 / 1.0$, flow rate $1.0 \mathrm{~mL} / \mathrm{min}, \lambda=254 \mathrm{~nm}) \mathrm{t}$ (major) $=14.050 \mathrm{~min}, \mathrm{t}$ (minor) $=8.758 \mathrm{~min}) .[\alpha]_{\mathrm{D}}^{25}=+26.8\left(\mathrm{CH}_{2} \mathrm{Cl}_{2}, c=0.19\right) .{ }^{1} \mathrm{H}-\mathrm{NMR}\left(300 \mathrm{MHz}, \mathrm{CDCl}_{3}\right) \delta: 7.80(\mathrm{~d}, J=7.9$ $\mathrm{Hz}, 1 \mathrm{H}), 7.65-7.59(\mathrm{~m}, 1 \mathrm{H}), 7.43-7.35(\mathrm{~m}, 2 \mathrm{H}), 7.25-7.17(\mathrm{~m}, 1 \mathrm{H}), 6.98-6.89(\mathrm{~m}, 3 \mathrm{H}), 3.50-3.36(\mathrm{~m}, 2 \mathrm{H})$, $3.31(\mathrm{~d}, J=13.9 \mathrm{~Hz}, 1 \mathrm{H})$ and $2.99(\mathrm{~d}, J=13.9 \mathrm{~Hz}, 1 \mathrm{H}) \mathrm{ppm} .{ }^{13} \mathrm{C}-\mathrm{NMR}\left(126 \mathrm{MHz}, \mathrm{CDCl}_{3}\right) \delta: 199.66$, $162.49(\mathrm{~d}, J=246.1 \mathrm{~Hz}), 149.33,136.33,136.27(\mathrm{~d}, J=7.4 \mathrm{~Hz}), 133.26,129.79(\mathrm{~d}, J=8.4 \mathrm{~Hz}), 128.42$, $126.34,126.25(\mathrm{~d}, J=2.9 \mathrm{~Hz}), 125.07,121.09(\mathrm{q}, J=258.9 \mathrm{~Hz}), 117.40(\mathrm{~d}, J=21.6 \mathrm{~Hz}), 114.43(\mathrm{~d}, J=20.9$ $\mathrm{Hz}), 86.72,41.90$ and $35.58 \mathrm{ppm} .{ }^{19} \mathrm{~F}-\mathrm{NMR}\left(282 \mathrm{MHz}_{2} \mathrm{CDCl}_{3}\right) \delta:-51.75(\mathrm{~s}, 3 \mathrm{~F})$ and $-113.50--113.58(\mathrm{~m}$, 1F) ppm. IR (NaCl): $v=3070,2931,1732,1610,1489,1448,1265,1149$ and $785 \mathrm{~cm}^{-1}$. HRMS (ESI) $\mathrm{m} / z$ : $[\mathrm{M}+\mathrm{Na}]^{+}$Calcd. for $\mathrm{C}_{17} \mathrm{H}_{12} \mathrm{~F}_{4} \mathrm{NaO}_{2}+347.0666$; found 347.0672 .

(+)-2-([1,1'-Biphenyl]-4-ylmethyl)-2-(trifluoromethoxy)-2,3-dihydro-1H-inden-1-one ((+)-3af). The reaction was run according to the general procedure. Eluent $(n$-hexane/ethyl acetate $=15 / 1)$. Slightly yellow oil; $31.0 \mathrm{mg}$; $81 \%$ yield. The enantiomeric excess ( $41 \%$ ee) was determined by chiral HPLC using CHIRALPAK $^{\circledR}$ ID ( $n$-hexane/isopropanol $=99.0 / 1.0$, flow rate $\left.1.0 \mathrm{~mL} / \mathrm{min}, \lambda=254 \mathrm{~nm}\right) \mathrm{t}$ (major) $=$ $10.408 \mathrm{~min}, \mathrm{t}$ (minor) $=8.192 \mathrm{~min}) .[\alpha]_{\mathrm{D}}^{25}=+27.3\left(\mathrm{CH}_{2} \mathrm{Cl}_{2}, c=0.84\right) .{ }^{1} \mathrm{H}-\mathrm{NMR}\left(300 \mathrm{MHz}, \mathrm{CDCl}_{3}\right) \delta: 7.80$ $(\mathrm{d}, J=7.7 \mathrm{~Hz}, 1 \mathrm{H}), 7.62-7.31(\mathrm{~m}, 10 \mathrm{H}), 7.27-7.25(\mathrm{~m}, 2 \mathrm{H}), 3.47(\mathrm{~s}, 2 \mathrm{H}), 3.36(\mathrm{~d}, J=13.9 \mathrm{~Hz}, 1 \mathrm{H})$ and 3.05 $(\mathrm{d}, J=13.9 \mathrm{~Hz}, 1 \mathrm{H}) \mathrm{ppm} .{ }^{13} \mathrm{C}-\mathrm{NMR}\left(126 \mathrm{MHz}, \mathrm{CDCl}_{3}\right) \delta: 200.04,149.54,140.49,140.19,136.21,133.38$, $132.89,130.93,128.75,128.30,127.34,126.99,126.96,126.33,125.01,121.16$ (q, $J=258.8 \mathrm{~Hz}), 87.06,41.88$ and $35.61 \mathrm{ppm} .{ }^{19} \mathrm{~F}-\mathrm{NMR}\left(282 \mathrm{MHz}, \mathrm{CDCl}_{3}\right) \delta:-51.64(\mathrm{~s}, 3 \mathrm{~F}) \mathrm{ppm}$. IR $(\mathrm{NaCl}): v=3032,2937,1730$, 1608, 1487, 1265, 1201, 1151 and $748 \mathrm{~cm}^{-1}$. HRMS (ESI) $\mathrm{m} / z$ : $[\mathrm{M}+\mathrm{Na}]^{+}$Calcd. for $\mathrm{C}_{23} \mathrm{H}_{17} \mathrm{~F}_{3} \mathrm{NaO}_{2}{ }^{+}$ 405.1073; found 405.1077 .

(+)-2-(Naphthalen-2-ylmethyl)-2-(trifluoromethoxy)-2,3-dihydro-1H-inden-1-one ((+)-3ag). The reaction was run according to the general procedure. Eluent $(n$-hexane/ethyl acetate $=15 / 1)$. Slightly yellow oil; $30.3 \mathrm{mg}$; $85 \%$ yield. The enantiomeric excess ( $24 \%$ ee) was determined by chiral HPLC using CHIRALPAK ${ }^{\circledR}$ ID ( $n$-hexane/isopropanol $=99.5 / 0.5$, flow rate $\left.1.0 \mathrm{~mL} / \mathrm{min}, \lambda=254 \mathrm{~nm}\right) \mathrm{t}$ (major) $=$ $9.708 \mathrm{~min}, \mathrm{t}$ (minor) $=8.233 \mathrm{~min}) .[\alpha]_{\mathrm{D}}^{25}=+18.1\left(\mathrm{CH}_{2} \mathrm{Cl}_{2}, c=0.77\right) .{ }^{1} \mathrm{H}-\mathrm{NMR}\left(300 \mathrm{MHz}, \mathrm{CDCl}_{3}\right) \delta$ : 7.82-7.26 (m, 11H), 3.54-3.39 (m, 3H) and $3.16(\mathrm{~d}, J=13.9 \mathrm{~Hz}, 1 \mathrm{H}) \mathrm{ppm} .{ }^{13} \mathrm{C}-\mathrm{NMR}\left(126 \mathrm{MHz}, \mathrm{CDCl}_{3}\right) \delta$ : 200.07, 149.55, 136.21, 133.33, 133.10, 132.47, 131.57, 129.53, 128.39, 128.29, 127.98, 127.65, 127.56, 126.33, $126.14,125.95,125.02,121.16(\mathrm{q}, J=258.9 \mathrm{~Hz}), 87.15,42.26$ and $35.50 \mathrm{ppm} .{ }^{19} \mathrm{~F}-\mathrm{NMR}\left(282 \mathrm{MHz}, \mathrm{CDCl}_{3}\right)$ 
$\delta:-51.64(\mathrm{~s}, 3 \mathrm{~F}) \mathrm{ppm}$. IR (NaCl): $v=3057,2927,1730,1608,1509,1468,1263,1153,1162,1045$ and $742 \mathrm{~cm}^{-1}$. HRMS (ESI) $\mathrm{m} / z$ : $[\mathrm{M}+\mathrm{Na}]^{+}$Calcd. for $\mathrm{C}_{21} \mathrm{H}_{15} \mathrm{~F}_{3} \mathrm{NaO}_{2}{ }^{+}$379.0916; found 379.0917.

(+)-2-(3,5-di-tert-Butylbenzyl)-2-(trifluoromethoxy)-2,3-dihydro-1H-inden-1-one ((+)-3ah). The reaction was run according to the general procedure. Eluent (n-hexane/ethyl acetate $=20 / 1)$. Yellow oil; $33.1 \mathrm{mg}$; $79 \%$ yield. The enantiomeric excess ( $37 \%$ ee) was determined by chiral HPLC using CHIRALPAK ${ }^{\circledR}$ IF column ( $n$-hexane/TBME $=95.0 / 5.0$, flow rate $0.5 \mathrm{~mL} / \mathrm{min}, \lambda=254 \mathrm{~nm}$ ) $\mathrm{t}$ (major) $=12.842 \mathrm{~min}, \mathrm{t}$ (minor) $=11.972 \mathrm{~min}) \cdot[\alpha]_{\mathrm{D}}^{25}=+24.3\left(\mathrm{CH}_{2} \mathrm{Cl}_{2}, c=0.87\right) .{ }^{1} \mathrm{H}-\mathrm{NMR}\left(300 \mathrm{MHz}, \mathrm{CDCl}_{3}\right) \delta: 7.69(\mathrm{~d}, J=7.8 \mathrm{~Hz}, 1 \mathrm{H})$, $7.51(\mathrm{t}, J=7.5 \mathrm{~Hz}, 1 \mathrm{H}), 7.32-7.15(\mathrm{~m}, 3 \mathrm{H}), 6.94(\mathrm{~s}, 2 \mathrm{H}), 3.45(\mathrm{~s}, 2 \mathrm{H}), 3.30(\mathrm{~d}, J=13.3 \mathrm{~Hz}, 1 \mathrm{H}), 3.14(\mathrm{~d}$, $J=13.3 \mathrm{~Hz}, 1 \mathrm{H})$ and $1.22(\mathrm{~s}, 18 \mathrm{H}) \mathrm{ppm} .{ }^{13} \mathrm{C}-\mathrm{NMR}\left(126 \mathrm{MHz}, \mathrm{CDCl}_{3}\right) \delta: 200.38,150.55,149.71,135.88$, 133.85, 132.40, 127.95, 125.99, 124.70, 124.59, 121.26 (q, $J=258.5 \mathrm{k} \mathrm{Hz}), 120.95,87.41,43.01,35.97,35.96$, 34.61 and $31.30 \mathrm{ppm} .{ }^{19} \mathrm{~F}-\mathrm{NMR}\left(282 \mathrm{MHz}, \mathrm{CDCl}_{3}\right) \delta:-51.56$ (s, 3F) ppm. IR (NaCl): $v=2961,2867$, $2359,2336,1730,1465,1368,1263,1199,1153$ and $830,720 \mathrm{~cm}^{-1}$. MS (ESI): $m / z 436\left[\mathrm{M}+\mathrm{NH}_{4}\right]^{+}$.

(+)-2-Benzyl-5-bromo-2-(trifluoromethoxy)-2,3-dihydro-1H-inden-1-one ((+)-3ba). The reaction was run according to the general procedure. Eluent $(n$-hexane/ethyl acetate $=15 / 1)$. Slightly yellow solid; 35.8 $\mathrm{mg} ; 93 \%$ yield. The enantiomeric excess (57\% ee) was determined by chiral HPLC using CHIRALCEL ${ }^{\circledR}$ OJ-H ( $n$-hexane/isopropanol $=99.0 / 1.0$, flow rate $1.0 \mathrm{~mL} / \mathrm{min}, \lambda=254 \mathrm{~nm}) \mathrm{t}$ (major) $=13.833 \mathrm{~min}$, $\mathrm{t}($ minor $)=18.725 \mathrm{~min}) . \quad[\alpha]_{\mathrm{D}}^{25}=+12.5\left(\mathrm{CH}_{2} \mathrm{Cl}_{2}, c=0.31\right) .{ }^{1} \mathrm{H}-\mathrm{NMR}\left(300 \mathrm{MHz}, \mathrm{CDCl}_{3}\right) \delta: 7.63(\mathrm{~d}$, $J=8.4 \mathrm{~Hz}, 1 \mathrm{H}), 7.53-7.51(\mathrm{~m}, 2 \mathrm{H}), 7.32-7.06(\mathrm{~m}, 5 \mathrm{H}), 3.41(\mathrm{~s}, 2 \mathrm{H}), 3.30(\mathrm{~d}, J=13.8 \mathrm{~Hz}, 1 \mathrm{H})$ and $3.02(\mathrm{~d}$, $J=13.8 \mathrm{~Hz}, 1 \mathrm{H}) \mathrm{ppm} .{ }^{13} \mathrm{C}-\mathrm{NMR}\left(126 \mathrm{MHz}, \mathrm{CDCl}_{3}\right) \delta: 198.92,151.00,133.42,132.26,131.99,131.66$, $130.47,129.58,128.43,127.55,126.09,121.09(\mathrm{q}, J=259.1 \mathrm{~Hz}), 86.77,42.15$ and $35.21 \mathrm{ppm} .{ }^{19} \mathrm{~F}-\mathrm{NMR}(282$ $\left.\left.\mathrm{MHz}, \mathrm{CDCl}_{3}\right)\right) \delta:-51.71(\mathrm{~s}, 3 \mathrm{~F}) \mathrm{ppm}$. IR (NaCl): $v=3031,2929,1734,1263,1203,1151,1058,704$ and $573 \mathrm{~cm}^{-1}$. HRMS (ESI) $\mathrm{m} / \mathrm{z}$ : $[\mathrm{M}+\mathrm{Na}]^{+}$Calcd. for $\mathrm{C}_{17} \mathrm{H}_{12} \mathrm{BrF}_{3} \mathrm{NaO}_{2}{ }^{+}$406.9865; found 406.9864. m.p.: $53.6-55.8^{\circ} \mathrm{C}$.

(+)-2-Benzyl-5-fluoro-2-(trifluoromethoxy)-2,3-dihydro-1H-inden-1-one ((+)-3ca). The reaction was run according to the general procedure. Eluent $(n$-hexane/ethyl acetate $=15 / 1)$. Slightly yellow oil; $23.0 \mathrm{mg}$; $71 \%$ yield. The enantiomeric excess $\left(51 \%\right.$ ee) was determined by chiral HPLC using CHIRALCEL ${ }^{\circledR}$ OJ-H ( $n$-hexane/isopropanol $=99.0 / 1.0$, flow rate $1.0 \mathrm{~mL} / \mathrm{min}, \lambda=254 \mathrm{~nm}) \mathrm{t}$ (major) $=28.833 \mathrm{~min}, \mathrm{t}$ (minor) $=27.167 \mathrm{~min}) .[\alpha]_{\mathrm{D}}^{25}=+27.0\left(\mathrm{CH}_{2} \mathrm{Cl}_{2}, c=0.77\right) .{ }^{1} \mathrm{H}-\mathrm{NMR}\left(300 \mathrm{MHz}, \mathrm{CDCl}_{3}\right) \delta: 7.81-7.76(\mathrm{~m}$, $1 \mathrm{H}), 7.23-7.17(\mathrm{~m}, 5 \mathrm{H}), 7.07(\mathrm{t}, J=8.8 \mathrm{~Hz}, 1 \mathrm{H}), 6.99(\mathrm{~d}, J=8.2 \mathrm{~Hz}, 1 \mathrm{H}), 3.42(\mathrm{~s}, 2 \mathrm{H}), 3.31(\mathrm{~d}, J=13.8 \mathrm{~Hz}$, $1 \mathrm{H})$ and $3.05(\mathrm{~d}, J=13.8 \mathrm{~Hz}, 1 \mathrm{H}) \mathrm{ppm} .{ }^{13} \mathrm{C}-\mathrm{NMR}\left(126 \mathrm{MHz} \mathrm{CDCl}_{3}\right) \delta: 198.16,167.78(\mathrm{~d}, J=259.1 \mathrm{~Hz})$, $152.48(\mathrm{~d}, J=10.5 \mathrm{~Hz}), 133.47,130.46,129.94(\mathrm{~d}, J=1.9 \mathrm{~Hz}), 128.39,127.51,127.48(\mathrm{~d}, J=10.5 \mathrm{~Hz}), 121.12$ $(\mathrm{q}, J=259.0 \mathrm{~Hz}), 116.73(\mathrm{~d}, J=23.7 \mathrm{~Hz}), 113.09(\mathrm{~d}, J=22.7 \mathrm{~Hz}), 86.97,42.20$ and $35.58 \mathrm{ppm} .{ }^{19} \mathrm{~F}-\mathrm{NMR}$ $\left(282 \mathrm{MHz}, \mathrm{CDCl}_{3}\right) \delta:-51.72(\mathrm{~s}, 3 \mathrm{~F})$ and $-99.80(\mathrm{q}, J=7.1 \mathrm{~Hz}, 1 \mathrm{~F}) \mathrm{ppm}$. IR $(\mathrm{NaCl}): v=3028,2929,1734$, 1616, 1595, 1263, 1200, 1151 and $702 \mathrm{~cm}^{-1}$. HRMS (ESI) $\mathrm{m} / z$ : $[\mathrm{M}+\mathrm{Na}]^{+}$Calcd. for $\mathrm{C}_{17} \mathrm{H}_{12} \mathrm{~F}_{4} \mathrm{NaO}_{2}{ }^{+}$ 347.0666; found 347.0672.

(+)-2-Benzyl-6-fluoro-2-(trifluoromethoxy)-2,3-dihydro-1H-inden-1-one ((+)-3da). The reaction was run according to the general procedure. Eluent ( $n$-hexane/ethyl acetate $=15 / 1)$. Slightly yellow oil; $26.6 \mathrm{mg}$; $82 \%$ yield. The enantiomeric excess ( $56 \%$ ee) was determined by chiral HPLC using CHIRALCEL ${ }^{\circledR}$ OJ-H ( $n$-hexane/isopropanol $=99.0 / 1.0$, flow rate $1.0 \mathrm{~mL} / \mathrm{min}, \lambda=254 \mathrm{~nm}) \mathrm{t}$ (major) $=13.792 \mathrm{~min}$, $\mathrm{t}$ (minor) $=9.725 \mathrm{~min}) .[\alpha]_{\mathrm{D}}^{25}=+22.2\left(\mathrm{CH}_{2} \mathrm{Cl}_{2}, c=0.80\right) .{ }^{1} \mathrm{H}-\mathrm{NMR}\left(300 \mathrm{MHz}, \mathrm{CDCl}_{3}\right) \delta: 7.40(\mathrm{~d}$, $J=7.0 \mathrm{~Hz}, 1 \mathrm{H}), 7.29(\mathrm{~d}, J=5.1 \mathrm{~Hz}, 2 \mathrm{H}), 7.23-7.14(\mathrm{~m}, 5 \mathrm{H}), 3.40(\mathrm{~s}, 2 \mathrm{H}), 3.30(\mathrm{~d}, J=13.8 \mathrm{~Hz}, 1 \mathrm{H})$ and $3.05(\mathrm{~d}, J=13.7 \mathrm{~Hz}, 1 \mathrm{H}) \mathrm{ppm} .{ }^{13} \mathrm{C}-\mathrm{NMR}\left(126 \mathrm{MHz}, \mathrm{CDCl}_{3}\right) \delta: 199.35,162.43(\mathrm{~d}, J=249.8 \mathrm{~Hz}), 145.02$ $(\mathrm{d}, J=2.3 \mathrm{~Hz}), 135.14(\mathrm{~d}, J=7.5 \mathrm{~Hz}), 133.45,130.44,128.38,127.84(\mathrm{~d}, J=7.8 \mathrm{~Hz}), 127.51,123.92(\mathrm{~d}$, $J=23.7 \mathrm{~Hz}), 121.12(\mathrm{q}, J=258.9 \mathrm{H} \mathrm{z}), 110.69(\mathrm{~d}, J=22.2 \mathrm{~Hz}), 87.49,42.27$ and $35.10 \mathrm{ppm} .{ }^{19} \mathrm{~F}-\mathrm{NMR}$ $\left(282 \mathrm{MHz}, \mathrm{CDCl}_{3}\right) \delta:-51.73(\mathrm{~s}, 3 \mathrm{~F})$ and $-112.86(\mathrm{q}, J=6.9 \mathrm{~Hz}, 1 \mathrm{~F}) \mathrm{ppm}$. IR $(\mathrm{NaCl}): v=3033,2947,1736$, $1614,1489,1265,1200,1155,775$ and $702 \mathrm{~cm}^{-1}$. HRMS (ESI) $\mathrm{m} / \mathrm{z}:[\mathrm{M}+\mathrm{Na}]^{+}$Calcd. for $\mathrm{C}_{17} \mathrm{H}_{12} \mathrm{~F}_{4} \mathrm{NaO}_{2}{ }^{+}$ 347.0666; found 347.0671. 
(+)-2-Benzyl-6-methyl-2-(trifluoromethoxy)-2,3-dihydro-1H-inden-1-one $((+)-3 e a)$. The reaction was run according to the general procedure. Eluent ( $n$-hexane/ethyl acetate $=15 / 1)$. Slightly yellow oil; $27.2 \mathrm{mg}$; $85 \%$ yield. The enantiomeric excess $\left(29 \%\right.$ ee) was determined by chiral HPLC using CHIRALCEL ${ }^{\circledR}$ $\mathrm{OJ}-\mathrm{H}$ ( $n$-hexane/isopropanol $=99.0 / 1.0$, flow rate $1.0 \mathrm{~mL} / \mathrm{min}, \lambda=254 \mathrm{~nm}) \mathrm{t}$ (major) $=17.217 \mathrm{~min}, \mathrm{t}$ $($ minor $)=8.958 \mathrm{~min}) .[\alpha]_{\mathrm{D}}^{25}=+20.0\left(\mathrm{CH}_{2} \mathrm{Cl}_{2}, c=0.82\right) .{ }^{1} \mathrm{H}-\mathrm{NMR}\left(300 \mathrm{MHz}, \mathrm{CDCl}_{3}\right) \delta: 7.58(\mathrm{~s}, 1 \mathrm{H}), 7.41$ $(\mathrm{d}, J=7.8 \mathrm{~Hz}, 1 \mathrm{H}), 7.26-7.17(\mathrm{~m}, 6 \mathrm{H}), 3.37(\mathrm{~s}, 2 \mathrm{H}), 3.30(\mathrm{~d}, J=13.8 \mathrm{~Hz}, 1 \mathrm{H}), 2.98(\mathrm{~d}, J=13.9 \mathrm{~Hz}, 1 \mathrm{H})$ and $2.38(\mathrm{~s}, 3 \mathrm{H}) \mathrm{ppm} .{ }^{13} \mathrm{C}-\mathrm{NMR}\left(126 \mathrm{MHz}, \mathrm{CDCl}_{3}\right) \delta: 200.12,146.90,138.35,137.45,133.98,133.47,130.54$, $128.29,127.33,125.98,124.84,121.14(q, J=258.5 \mathrm{~Hz}), 87.37,42.22,35.14$ and 21.11 ppm. ${ }^{19} \mathrm{~F}-\mathrm{NMR}(282$ $\left.\mathrm{MHz}_{2} \mathrm{CDCl}_{3}\right) \delta:-51.68$ (s, 3F) ppm. IR (NaCl): $v=3034,2929,1727,1495,1265,1153$ and $702 \mathrm{~cm}^{-1}$. HRMS (ESI) $m / z$ : $[\mathrm{M}+\mathrm{Na}]^{+}$Calcd. for $\mathrm{C}_{18} \mathrm{H}_{15} \mathrm{~F}_{3} \mathrm{NaO}_{2}{ }^{+}$343.0916; found 343.0913.

(+)-2-Benzyl-6-methoxy-2-(trifluoromethoxy)-2,3-dihydro-1H-inden-1-one ((+)-3fa). The reaction was run according to the general procedure. Eluent ( $n$-hexane/ethyl acetate $=10 / 1$ ). Yellow solid; $30.9 \mathrm{mg} ; 92 \%$ yield. The enantiomeric excess (33\% ee) was determined by chiral HPLC using CHIRALCEL ${ }^{\circledR}$ OJ-H ( $n$-hexane/isopropanol $=99.0 / 1.0$, flow rate $1.0 \mathrm{~mL} / \mathrm{min}, \lambda=254 \mathrm{~nm}) \mathrm{t}$ (major) $=26.942 \mathrm{~min}, \mathrm{t}($ minor $)=$ $16.517 \mathrm{~min}) .[\alpha]_{\mathrm{D}}^{25}=+29.2\left(\mathrm{CH}_{2} \mathrm{Cl}_{2}, c=0.67\right) .{ }^{1} \mathrm{H}-\mathrm{NMR}\left(300 \mathrm{MHz}, \mathrm{CDCl}_{3}\right) \delta: 7.26-7.17(\mathrm{~m}, 8 \mathrm{H}), 3.83$ $(\mathrm{s}, 3 \mathrm{H}), 3.35(\mathrm{~s}, 2 \mathrm{H}), 3.30(\mathrm{~d}, J=13.8 \mathrm{~Hz}, 1 \mathrm{H})$ and $3.00(\mathrm{~d}, J=13.8 \mathrm{~Hz}, 1 \mathrm{H}) \mathrm{ppm} .{ }^{13} \mathrm{C}-\mathrm{NMR}(126 \mathrm{MHz}$, $\left.\mathrm{CDCl}_{3}\right) \delta:$ 200.13, 159.80, 142.42, 134.48, 133.91, 130.50, 128.29, 127.37, 127.09, 125.69, $121.14(\mathrm{q}, \mathrm{J}=258.8$ $\mathrm{Hz}), 105.79,87.65,55.64,42.33$ and 34.87 ppm. ${ }^{19} \mathrm{~F}-\mathrm{NMR}\left(282 \mathrm{MHz}, \mathrm{CDCl}_{3}\right) \delta:-51.73$ (s, 3F) ppm. IR $(\mathrm{NaCl}): v=3032,2945,1728,1618,1493,1435,1271,1028,769$ and $702 \mathrm{~cm}^{-1}$. HRMS (ESI) $m / z:[\mathrm{M}+$ $\mathrm{Na}]^{+}$Calcd. for $\mathrm{C}_{18} \mathrm{H}_{15} \mathrm{~F}_{3} \mathrm{NaO}_{3}{ }^{+}$359.0866; found 359.0869. m.p.: 93.5-95.7 ${ }^{\circ} \mathrm{C}$.

(+)-2-Benzyl-5,6-dimethoxy-2-(trifluoromethoxy)-2,3-dihydro-1H-inden-1-one ((+)-3ga). The reaction was run according to the general procedure. Eluent ( $n$-hexane/ethyl acetate $=5 / 1$ ). Orange solid; $24.9 \mathrm{mg}$; $68 \%$ yield. The enantiomeric excess $(13 \%$ ee) was determined by chiral HPLC using a series of CHIRALPAK $^{\circledR}$ IF and CHIRALPAK ${ }^{\circledR}$ IA-3 ( $n$-hexane/isopropanol $=90.0 / 10.0$, flow rate $1.0 \mathrm{~mL} / \mathrm{min}, \lambda$ $=254 \mathrm{~nm}) \mathrm{t}$ (major) $=15.475 \mathrm{~min}, \mathrm{t}($ minor $)=23.808 \mathrm{~min}) .[\alpha]_{\mathrm{D}}^{25}=-4.5\left(\mathrm{CH}_{2} \mathrm{Cl}_{2}, c=0.83\right) .{ }^{1} \mathrm{H}-\mathrm{NMR}$ $\left(300 \mathrm{MHz}, \mathrm{CDCl}_{3}\right)$ 8: 7.27-7.18 (m, 6H), $6.72(\mathrm{~s}, 1 \mathrm{H}), 3.93(\mathrm{~s}, 3 \mathrm{H}), 3.90(\mathrm{~s}, 3 \mathrm{H}), 3.34-3.29(\mathrm{~m}, 3 \mathrm{H})$ and 3.02 $(\mathrm{d}, J=13.8 \mathrm{~Hz}, 1 \mathrm{H})$ ppm. ${ }^{13} \mathrm{C}-\mathrm{NMR}\left(126 \mathrm{MHz}, \mathrm{CDCl}_{3}\right) \delta: 198.42,156.63,149.97,145.30,134.08,130.51$, 128.27, 127.32, 126.22, 121.16 (q, $J=258.5 \mathrm{~Hz}), 107.02,104.96,87.37,56.34,56.14,42.31$ and 35.24 ppm. ${ }^{19} \mathrm{~F}-\mathrm{NMR}\left(282 \mathrm{MHz}, \mathrm{CDCl}_{3}\right) \delta:-51.72$ (s, 3F) ppm. IR $(\mathrm{NaCl}): v=3030,2939,1716,1591,1502,1268$, 1196, 1146, 782 and $702 \mathrm{~cm}^{-1}$. HRMS (ESI) $\mathrm{m} / \mathrm{z}:[\mathrm{M}+\mathrm{Na}]^{+}$Calcd. for $\mathrm{C}_{19} \mathrm{H}_{17} \mathrm{~F}_{3} \mathrm{NaO}_{4}{ }^{+} 389.0971$; found 389.0976. m.p.: $101.4-105.2^{\circ} \mathrm{C}$.

(+)-2-Benzyl-2-(trifluoromethoxy)-3,4-dihydronaphthalen-1(2H)-one $((+)-3 h a)$. The reaction was run according to the general procedure. Eluent ( $n$-hexane/ethyl acetate $=10 / 1)$. Slightly yellow oil; $16.3 \mathrm{mg}$; $51 \%$ yield. The enantiomeric excess $\left(8 \%\right.$ ee) was determined by chiral HPLC using CHIRALCEL ${ }^{\circledR}$ OJ-H (n-hexane/isopropanol $=99.0 / 1.0$, flow rate $1.0 \mathrm{~mL} / \mathrm{min}, \lambda=254 \mathrm{~nm}$ ) $\mathrm{t}$ (major) $=22.442 \mathrm{~min}, \mathrm{t}$ (minor) $=8.583 \mathrm{~min}) .[\alpha]_{\mathrm{D}}^{25}=-0.1\left(\mathrm{CH}_{2} \mathrm{Cl}_{2}, c=0.30\right) .{ }^{1} \mathrm{H}-\mathrm{NMR}\left(300 \mathrm{MHz}, \mathrm{CDCl}_{3}\right) \delta: 8.13(\mathrm{dd}, \mathrm{J}=7.8$, $1.5 \mathrm{~Hz}, 1 \mathrm{H}), 7.55(\mathrm{td}, J=7.5,1.5 \mathrm{~Hz}, 1 \mathrm{H}), 7.38(\mathrm{t}, J=7.5 \mathrm{~Hz}, 1 \mathrm{H}), 7.35-7.26(\mathrm{~m}, 6 \mathrm{H}), 3.29-3.16(\mathrm{~m}, 2 \mathrm{H})$, $3.11(\mathrm{t}, J=6.3 \mathrm{~Hz}, 2 \mathrm{H}), 2.49(\mathrm{dt}, J=14.1,7.1 \mathrm{~Hz}, 1 \mathrm{H})$ and $2.14(\mathrm{dt}, J=13.7,5.1 \mathrm{~Hz}, 1 \mathrm{H}) \mathrm{ppm} .{ }^{13} \mathrm{C}-\mathrm{NMR}$ $\left(126 \mathrm{MHz}_{,} \mathrm{CDCl}_{3}\right) \delta: 192.65,142.15,134.27,134.25,130.68,130.63,128.71,128.68,128.38,127.33,127.25$, $121.09(\mathrm{q}, J=258.4 \mathrm{~Hz}), 85.72,39.78,30.41$ and $26.01 \mathrm{ppm} .{ }^{19} \mathrm{~F}-\mathrm{NMR}\left(282 \mathrm{MHz}, \mathrm{CDCl}_{3}\right) \delta:-50.57(\mathrm{~s}, 3 \mathrm{~F})$ ppm. IR $(\mathrm{NaCl}): v=3032,2949,1699,1603,1454,1273,1201,1146,908$ and $706 \mathrm{~cm}^{-1}$. HRMS (ESI) $m / z$ : $[\mathrm{M}+\mathrm{Na}]^{+}$Calcd. for $\mathrm{C}_{18} \mathrm{H}_{15} \mathrm{~F}_{3} \mathrm{NaO}_{2}{ }^{+}$343.0916; found 343.0922.

(R)-2-Allyl-2-(trifluoromethoxy)-2,3-dihydro-1H-inden-1-one (3ai). The reaction was run according to the general procedure, and the product is consistent with previously reported characterization data [46]. Eluent $(n$-hexane/ethyl acetate $=15 / 1)$. Colorless oil; $11.0 \mathrm{mg} ; 43 \%$ yield. The enantiomeric excess $(68 \%$ ee) was determined by chiral HPLC using a CHIRALCEL ${ }^{\circledR}$ OJ-H column ( $n$-hexane/isopropanol = 98.0/2.0, flow rate $0.5 \mathrm{~mL} / \mathrm{min}, \lambda=254 \mathrm{~nm}$ ) $\mathrm{t}$ (major) $=11.167 \mathrm{~min}, \mathrm{t}$ (minor) $=9.975 \mathrm{~min}) .[\alpha]_{\mathrm{D}}^{25}=$ $+16.5\left(\mathrm{CHCl}_{3}, c=0.37\right) .{ }^{1} \mathrm{H}-\mathrm{NMR}\left(300 \mathrm{MHz} \mathrm{CDCl}_{3}\right) \delta: 7.82(\mathrm{~d}, J=7.7 \mathrm{~Hz}, 1 \mathrm{H}), 7.68(\mathrm{t}, J=7.5 \mathrm{~Hz}, 1 \mathrm{H})$, 
7.46-7.42 (m, 2H), $5.67(\mathrm{ddt}, J=17.3,10.4,7.2 \mathrm{~Hz}, 1 \mathrm{H}), 5.19-5.11(\mathrm{~m}, 2 \mathrm{H}), 3.54(\mathrm{~d}, J=17.8 \mathrm{~Hz}, 1 \mathrm{H})$, $3.41(\mathrm{~d}, J=17.8 \mathrm{~Hz}, 1 \mathrm{H}), 2.75(\mathrm{dd}, J=14.0,6.7 \mathrm{~Hz}, 1 \mathrm{H}), 2.55(\mathrm{dd}, J=14.0,7.5 \mathrm{~Hz}, 1 \mathrm{H}) \mathrm{ppm} .{ }^{19} \mathrm{~F}-\mathrm{NMR}$ $\left(282 \mathrm{MHz}, \mathrm{CDCl}_{3}\right) \delta:-51.90(\mathrm{~s}, 3 \mathrm{~F}) \mathrm{ppm}$.

(R)-2-Allyl-5-fluoro-2-(trifluoromethoxy)-2,3-dihydro-1H-inden-1-one (3ci). The reaction was run according to the general procedure, and the product is consistent with previously reported characterization data. [46] Eluent ( $n$-hexane/ethyl acetate $=20 / 1$ ). Colorless oil; $9.6 \mathrm{mg} ; 35 \%$ yield. The enantiomeric excess $\left(70 \%\right.$ ee) was determined by chiral HPLC using CHIRALPAK ${ }^{\circledR}$ IF column $(n$-hexane/TBME $=$ 90.0/10.0, flow rate $0.5 \mathrm{~mL} / \mathrm{min}, \lambda=254 \mathrm{~nm}) \mathrm{t}$ (major) $=15.192 \mathrm{~min}, \mathrm{t}($ minor $)=13.042 \mathrm{~min}) .[\alpha]_{\mathrm{D}}^{25}=$ $+19.7\left(\mathrm{CH}_{2} \mathrm{Cl}_{2}, c=0.31\right) .{ }^{1} \mathrm{H}-\mathrm{NMR}\left(300 \mathrm{MHz}, \mathrm{CDCl}_{3}\right) \delta: 7.85(\mathrm{~d}, J=4.5 \mathrm{~Hz}, 1 \mathrm{H}), 7.18-7.12(\mathrm{~m}, 2 \mathrm{H})$, $5.67-5.60(\mathrm{~m}, 1 \mathrm{H}), 5.21-5.12(\mathrm{~m}, 2 \mathrm{H}), 3.53(\mathrm{~d}, J=18.0 \mathrm{~Hz}, 1 \mathrm{H}), 3.40(\mathrm{~d}, J=18.1 \mathrm{~Hz}, 1 \mathrm{H}), 2.79-2.72(\mathrm{~m}, 1 \mathrm{H})$, 2.60-2.52 (m, 1H) ppm. ${ }^{19} \mathrm{~F}-\mathrm{NMR}\left(282 \mathrm{MHz}, \mathrm{CDCl}_{3}\right) \delta:-51.95(\mathrm{~s}, 3 \mathrm{~F})$ and $-99.62--99.73(\mathrm{~m}, 1 \mathrm{~F}) \mathrm{ppm}$.

(R)-2-Allyl-6-methyl-2-(trifluoromethoxy)-2,3-dihydro-1H-inden-1-one (3ei). The reaction was run according to the general procedure, and the product was consistent with previously reported characterization data [46]. Eluent ( $n$-hexane/ethyl acetate $=15 / 1$ ). Colorless oil; $14.0 \mathrm{mg} ; 52 \%$ yield. The enantiomeric excess $\left(45 \%\right.$ ee) was determined by chiral HPLC using CHIRALPAK ${ }^{\circledR}$ IF column ( $n$-hexane/TBME $=$ $90.0 / 10.0$, flow rate $0.5 \mathrm{~mL} / \mathrm{min}, \lambda=254 \mathrm{~nm}) \mathrm{t}$ (major) $=15.992 \mathrm{~min}, \mathrm{t}($ minor $)=13.083 \mathrm{~min}) .[\alpha]_{\mathrm{D}}^{25}=$ $+16.2\left(\mathrm{CH}_{2} \mathrm{Cl}_{2}, \mathrm{c}=0.46\right) .{ }^{1} \mathrm{H}-\mathrm{NMR}\left(300 \mathrm{MHz}, \mathrm{CDCl}_{3}\right) \delta: 7.61(\mathrm{~s}, 1 \mathrm{H}), 7.49(\mathrm{~d}, J=7.9 \mathrm{~Hz}, 1 \mathrm{H}), 7.32(\mathrm{~d}, J=$ $7.7 \mathrm{~Hz}, 1 \mathrm{H}), 5.70-5.59(\mathrm{~m}, 1 \mathrm{H}), 5.18-5.10(\mathrm{~m}, 2 \mathrm{H}), 3.48(\mathrm{~d}, J=17.7 \mathrm{~Hz}, 1 \mathrm{H}), 3.35(\mathrm{~d}, J=17.5 \mathrm{~Hz}, 1 \mathrm{H})$, 2.76-2.69 (m, 1H), 2.57-2.50 (m, 1H) and $2.41(\mathrm{~s}, 3 \mathrm{H}) \mathrm{ppm} .{ }^{19} \mathrm{~F}-\mathrm{NMR}\left(282 \mathrm{MHz}, \mathrm{CDCl}_{3}\right) \delta:-51.90(\mathrm{~s}$, 3F) ppm.

\section{Conclusions}

In conclusion, we disclose the organo-catalytic enantioselective benzylation reaction of $\alpha-\mathrm{OCF}_{3}$-indanones 1. $\alpha$-Benzyl- $\alpha-\mathrm{OCF}_{3}$-indanones 3 was synthesized in good to high yield with moderate enantioselectivity, up to $57 \%$ ee, and both enantiomers of 3 could be accessed by the selection of chiral PTC, CN-4 or CD-4. The method was extended to the enantioselective allylation of $\mathbf{1}$, and both enantiomers of $\alpha$-allyl- $\alpha-\mathrm{OCF}_{3}$-indanones were also obtained in moderate yield with good ee, as much as $76 \%$ ee. To our knowledge, this is the first example of the asymmetric synthesis of trifluoromethoxylated compounds with a stereogenic $\mathrm{OCF}_{3}$-carbon center, without the use of transition metals. Extension of this methodology to other $\mathrm{OCF}_{3}$ ketones is underway, and will be reported in due course [48].

Supplementary Materials: The following are available online, ${ }^{1} \mathrm{H},{ }^{13} \mathrm{C}$ and ${ }^{19} \mathrm{~F}-\mathrm{NMR}$ spectra for desired compounds 3 and HPLC data for desired compounds 3.

Author Contributions: N.S. conceived and designed the experiments and directed the project; M.M. Y.L. and Z.Z. performed the experiments; Y.L. analyzed the data; N.S. and Y.L. wrote the paper.

Funding: This work was supported by JSPS KAKENHI grants JP 18H02553 (KIBAN B) and JP 18H04401 (Middle Molecular Strategy).

Conflicts of Interest: The authors declare no conflicts of interest.

\section{References and Note}

1. Gillis, E.P.; Eastman, K.J.; Hill, M.D.; Donnelly, D.J.; Meanwell, N.A. Applications of fluorine in medicinal chemistry. J. Med. Chem. 2015, 58, 8315-8359. [CrossRef] [PubMed]

2. Berger, R.; Resnati, G.; Metrangolo, P.; Weber, E.; Hulliger, J. Organic fluorine compounds: A great opportunity for enhanced materials properties. Chem. Soc. Rev. 2011, 40, 3496-3508. [CrossRef] [PubMed]

3. Purser, S.; Moore, P.R.; Swallow, S.; Gouverneur, V. Fluorine in medicinal chemistry. Chem. Soc. Rev. 2008, 37, 320-330. [CrossRef] [PubMed]

4. Isanbor, C.; O'Hagan, D. Fluorine in medicinal chemistry: A review of anti-cancer agents. J. Fluor. Chem. 2006, 127, 303-319. [CrossRef] 
5. Müller, K.; Faeh, C.; Diederich, F. Fluorine in pharmaceuticals: Looking beyond intuition. Science 2007, 317, 1881-1886. [CrossRef] [PubMed]

6. Meanwell, N.A. Fluorine and fluorinated motifs in the design and application of bioisosteres for drug design. J. Med. Chem. 2018, 61, 5822-5880. [CrossRef] [PubMed]

7. Kawai, H.; Shibata, N. Asymmetric Synthesis of Agrochemically Attractive Trifluoromethylated Dihydroazoles and Related Compounds under Organocatalysis. Chem. Rec. 2014, 14, 1024-1040. [CrossRef]

8. Shibata, N.; Mizuta, S.; Kawai, H. Recent advances in enantioselective trifluoromethylation reactions. Tetrahedron Asymmetry 2008, 19, 2633-2644. [CrossRef]

9. Zhu, Y.; Han, J.; Wang, J.; Shibata, N.; Sodeoka, M.; Soloshonok, V.A.; Coelho, J.A.; Toste, F.D. Modern approaches for asymmetric construction of carbon-fluorine quaternary stereogenic centers: Synthetic challenges and pharmaceutical needs. Chem. Rev. 2018, 118, 3887-3964. [CrossRef]

10. Mori, S.; Shibata, N. Synthesis and application of trifluoroethoxy-substituted phthalocyanines and subphthalocyanines. Beilstein J. Org. Chem. 2017, 13, 2273-2296. [CrossRef]

11. Smart, B.E. Fluorine substituent effects (on bioactivity). J. Fluor. Chem. 2001, 109, 3-11. [CrossRef]

12. $\mathrm{Xu}, \mathrm{X} . \mathrm{H}$.; Matsuzaki, K.; Shibata, N. Synthetic methods for compounds having $\mathrm{CF}_{3}-\mathrm{S}$ units on carbon by trifluoromethylation, trifluoromethylthiolation, triflylation, and related reactions. Chem. Rev. 2014, 115, 731-764. [CrossRef] [PubMed]

13. Zhou, Y.; Wang, J.; Gu, Z.; Wang, S.; Zhu, W.; Acenña, J.L.; Soloshonok, V.A.; Izawa, K.; Liu, H. Next generation of fluorine-containing pharmaceuticals, compounds currently in phase II-III clinical trials of major pharmaceutical companies: New structural trends and therapeutic areas. Chem. Rev. 2016, 116, 422-518. [CrossRef] [PubMed]

14. Shibata, N.; Ishimaru, T.; Nakamura, S.; Toru, T. New approaches to enantioselective fluorination: Cinchona alkaloids combinations and chiral ligands/metal complexes. J. Fluor. Chem. 2007, 128, 469-483. [CrossRef]

15. Shimizu, M.; Hiyama, T. Modern synthetic methods for fluorine-substituted target molecules. Angew. Chem. Int. Ed. 2005, 44, 214-231. [CrossRef] [PubMed]

16. Leroux, F.; Jeschke, P.; Schlosser, M. $\alpha$-Fluorinated ethers, thioethers, and amines: Anomerically biased species. Chem. Rev. 2005, 105, 827-856. [CrossRef] [PubMed]

17. Landelle, G.; Panossian, A.; R Leroux, F. Trifluoromethyl ethers and-thioethers as tools for Medicinal chemistry and drug discovery. Curr. Top. Med. Chem. 2014, 14, 941-951. [CrossRef] [PubMed]

18. Jeschke, P.; Baston, E.; Leroux, F.R. Alpha-fluorinated ethers as "exotic" entity in medicinal chemistry. Mini Rev. Med. Chem. 2007, 7, 1027-1034. [CrossRef]

19. Kirsch, P.; Bremer, M. Nematic liquid crystals for active matrix displays: Molecular design and synthesis. Angew. Chem. Int. Ed. 2000, 39, 4216-4235. [CrossRef]

20. Mamada, M.; Shima, H.; Yoneda, Y.; Shimano, T.; Yamada, N.; Kakita, K.; Machita, T.; Tanaka, Y.; Aotsuka, S.; Kumaki, D.; et al. A unique solution-processable n-type semiconductor material design for high-performance organic field-effect transistors. Chem. Mater. 2014, 27, 141-147. [CrossRef]

21. Coric, V.; Taskiran, S.; Pittenger, C.; Wasylink, S.; Mathalon, D.H.; Valentine, G.; Saksa, J.; Gueorguieva, R.; Sanacora, G.; Malison, R.T.; et al. Riluzole augmentation in treatment-resistant obsessive-compulsive disorder: An open-label trial. Biol. Psychiatry 2005, 58, 424-428. [CrossRef] [PubMed]

22. Diacon, A.H.; Dawson, R.; von Groote-Bidlingmaier, F.; Symons, G.; Venter, A.; Donald, P.R.; van Niekerk, C.; Everitt, D.; Winter, H.; Becker, P.; et al. 14-day bactericidal activity of PA-824, bedaquiline, pyrazinamide, and moxifloxacin combinations: A randomised trial. Lancet 2012, 380, 986-993. [CrossRef]

23. Henne, K.R.; Tran, T.B.; VandenBrink, B.M.; Rock, D.A.; Aidasani, D.K.; Subramanian, R.; Mason, A.K.; Stresser, D.M.; Teffera, Y.; Wong, S.G.; et al. Sequential metabolism of AMG 487, a novel CXCR3 antagonist, results in formation of quinone reactive metabolites that covalently modify CYP3A4 Cys239 and cause time-dependent inhibition of the enzyme. Drug Metab. Dispos. 2012, 40, 1429-1440. [CrossRef] [PubMed]

24. Leo, A.; Hansch, C.; Elkins, D. Partition coefficients and their uses. Chem. Rev. 1971, 71, 525-616. [CrossRef]

25. Hansch, C.; Leo, A.; Unger, S.H.; Kim, K.H.; Nikaitani, D.; Lien, E.J. Aromatic substituent constants for structure-activity correlations. J. Med. Chem. 1973, 16, 1207-1216. [CrossRef] [PubMed]

26. Hansch, C.; Leo, A.; Taft, R.W. A survey of Hammett substituent constants and resonance and field parameters. Chem. Rev. 1991, 91, 165-195. [CrossRef]

27. McClinton, M.A.; McClinton, D.A. Trifluoromethylations and related reactions in organic chemistry. Tetrahedron 1992, 48, 6555-6666. [CrossRef] 
28. Federsel, D.; Herrmann, A.; Christen, D.; Sander, S.; Willner, H.; Oberhammer, H. Structure and conformation of $\alpha, \alpha, \alpha$-trifluoroanisol, $\mathrm{C}_{6} \mathrm{H}_{5} \mathrm{OCF}_{3}$. J. Mol. Struct. 2001, 567, 127-136. [CrossRef]

29. Böhm, H.J.; Banner, D.; Bendels, S.; Kansy, M.; Kuhn, B.; Müller, K.; Obst-Sander, U.; Stahl, M. Fluorine in medicinal chemistry. ChemBioChem 2004, 5, 637-643. [CrossRef] [PubMed]

30. Farnham, W.B.; Smart, B.E.; Middleton, W.J.; Calabrese, J.C.; Dixon, D.A. Crystal and molecular structure of tris (dimethylamino) sulfonium trifluoromethoxide. Evidence for negative fluorine hyperconjugation. J. Am. Chem. Soc. 1985, 107, 4565-4567. [CrossRef]

31. Taylor, S.L.; Martin, J.C. Trifluoromethyl triflate: Synthesis and reactions. J. Org. Chem. 1987, 52, 4147-4156. [CrossRef]

32. Marrec, O.; Billard, T.; Vors, J.P.; Pazenok, S.; Langlois, B.R. A deeper insight into direct trifluoromethoxylation with trifluoromethyl triflate. J. Fluor. Chem. 2010, 131, 200-207. [CrossRef]

33. Sheppard, W.A. $\alpha$-Fluorinated Ethers. I. Aryl Fluoroalkyl Ethers1. J. Org. Chem. 1964, 29, 1-11. [CrossRef]

34. Marrec, O.; Billard, T.; Vors, J.P.; Pazenok, S.; Langlois, B.R. A New and Direct Trifluoromethoxylation of Aliphatic Substrates with 2,4-Dinitro (trifluoromethoxy) benzene. Adv. Synth. Catal. 2010, 352, 2831-2837. [CrossRef]

35. Huang, C.; Liang, T.; Harada, S.; Lee, E.; Ritter, T. Silver-mediated trifluoromethoxylation of aryl stannanes and arylboronic acids. J. Am. Chem. Soc. 2011, 133, 13308-13310. [CrossRef] [PubMed]

36. Chen, C.; Chen, P.; Liu, G. Palladium-catalyzed intramolecular aminotrifluoromethoxylation of alkenes. J. Am. Chem. Soc. 2015, 137, 15648-15651. [CrossRef]

37. Chen, C.; Luo, Y.; Fu, L.; Chen, P.; Lan, Y.; Liu, G. Palladium-Catalyzed Intermolecular Ditrifluoromethoxylation of Unactivated Alkenes: $\mathrm{CF}_{3} \mathrm{O}-\mathrm{Palladation}$ Initiated by Pd (IV). J. Am. Chem. Soc. 2018, 140, 1207-1210. [CrossRef]

38. Qi, X.; Chen, P.; Liu, G. Catalytic Oxidative Trifluoromethoxylation of Allylic C-H Bonds Using a Palladium Catalyst. Angew. Chem. Int. Ed. 2017, 56, 9517-9521. [CrossRef]

39. Zhou, M.; Ni, C.; Zeng, Y.; Hu, J. Trifluoromethyl benzoate: A versatile trifluoromethoxylation reagent. J. Am. Chem. Soc. 2018, 140, 6801-6805. [CrossRef]

40. Zheng, W.; Morales-Rivera, C.A.; Lee, J.W.; Liu, P.; Ngai, M.Y. Catalytic C-H Trifluoromethoxylation of Arenes and Heteroarenes. Angew. Chem. 2018, 130, 9793-9797. [CrossRef]

41. Yang, H.; Wang, F.; Jiang, X.; Zhou, Y.; Xu, X.; Tang, P. Silver-Promoted Oxidative Benzylic C-H Trifluoromethoxylation. Angew. Chem. Int. Ed. 2018, 57, 13266-13270. [CrossRef] [PubMed]

42. Jelier, B.J.; Tripet, P.F.; Pietrasiak, E.; Franzoni, I.; Jeschke, G.; Togni, A. Radical Trifluoromethoxylation of Arenes Triggered by a Visible-Light-Mediated N-O Bond Redox Fragmentation. Angew. Chem. Int. Ed. 2018, 57, 13784-13789. [CrossRef] [PubMed]

43. Guo, S.; Cong, F.; Guo, R.; Wang, L.; Tang, P. Asymmetric silver-catalysed intermolecular bromotrifluoromethoxylation of alkenes with a new trifluoromethoxylation reagent. Nat. Chem. 2017, 9, 546. [CrossRef] [PubMed]

44. Huang, W.; Wan, X.; Shen, Q. Enantioselective Construction of Trifluoromethoxylated Stereogenic Centers by a Nickel-Catalyzed Asymmetric Suzuki-Miyaura Coupling of Secondary Benzyl Bromides. Angew. Chem. Int. Ed. 2017, 56, 11986-11989. [CrossRef]

45. Kondo, H.; Maeno, M.; Hirano, K.; Shibata, N. Asymmetric synthesis of $\alpha$-trifluoromethoxy ketones with a tetrasubstituted $\alpha$-stereogenic centre via the palladium-catalyzed decarboxylative allylic alkylation of allyl enol carbonates. Chem. Commun. 2018, 54, 5522-5525. [CrossRef] [PubMed]

46. Kondo, H.; Maeno, M.; Sasaki, K.; Guo, M.; Hashimoto, M.; Shiro, M.; Shibata, N. Synthesis of chiral nonracemic $\alpha$-difluoromethylthio compounds with tetrasubstituted stereogenic centers via a palladium-catalyzed decarboxylative asymmetric allylic alkylation. Org. Lett. 2018, 20, 7044-7048. [CrossRef]

47. Chen, C.; Pflüger, P.M.; Chen, P.; Liu, G. Palladium (II)-Catalyzed Enantioselective Aminotrifluoromethoxylation of Unactivated Alkenes using $\mathrm{CsOCF}_{3}$ as a Trifluoromethoxide Source. Angew. Chem. Int. Ed. 2019, 58, 2392-2396. [CrossRef]

48. We attempted the alkylation reaction using aliphatic halides, but the no reaction was observed.

Sample Availability: Samples of the compounds 1a and 3aa are available from the authors. 